\title{
Improved Historical Analysis of Oceanic Total Precipitable Water*
}

\author{
THOMAS M. SMITH \\ NOAA/NESDIS/STAR, and Cooperative Institute for Climate and Satellites, Earth System Science Interdisciplinary \\ Center, University of Maryland, College Park, College Park, Maryland \\ PHILLIP A. ARKIN \\ Cooperative Institute for Climate and Satellites, Earth System Science Interdisciplinary Center, University of Maryland, \\ College Park, College Park, Maryland
}

(Manuscript received 28 August 2014, in final form 8 January 2015)

\begin{abstract}
The amount of water vapor in the atmosphere, total precipitable water (TPW), is an important part of the global water cycle, and a clearer understanding of ocean-area TPW is critical for understanding climate variations. This study uses satellite-period statistics and historical data to analyze monthly oceanic TPW beginning in the nineteenth century. Input data for analyzing the historical TPW includes outputs from an extended dynamic reanalysis and estimates of TPW based on historical sea surface temperature (SST). Methods are developed to optimally use the various inputs to produce an improved analysis. Cross-validation testing is used to guide analysis development. Some evaluation of the resulting analysis indicates several strong climate modes. A global mode indicates multidecadal increases in TPW since the nineteenth century, with strongest increases in the tropics and adjacent to land monsoon regions. Strongest multidecadal changes in the global mode are 1910-40 and since 1980. An ENSO mode for the extended period indicates a trend since the 1980s, opposite to the tendency in the global mode. There is no apparent multidecadal variation in the ENSO mode before 1980, suggesting that its multidecadal relationship with the global mode can change. Analysis of SST over the same period shows climate modes consistent with the TPW modes, and for the satellite period there are consistent variations in the satellite data, showing the strong link between SST and oceanic TPW.
\end{abstract}

\section{Introduction}

Since about $70 \%$ of the earth's surface is covered by oceans and most atmospheric water vapor forms from oceanic evaporation, the oceanic total precipitable water (TPW) is a critical component of the global hydrologic cycle (e.g., Wang and Lau 2006; Wentz et al. 2007; Hartmann et al. 2014). Thus, measuring and understanding changes in oceanic TPW is important for assessing changes in the global hydrologic cycle and the atmosphere's radiative balance (e.g., Mears et al. 2007;

\footnotetext{
* Supplemental information related to this paper is available at the Journals Online website: http://dx.doi.org/10.1175/JCLI-D-14-00601.s1.

Corresponding author address: Thomas Smith, NOAA/NESDIS/ STAR/SCSB and ESSIC/CICS, 5825 University Research Ct., Suite 4001, College Park, MD 20740.

E-mail: tom.smith@noaa.gov
}

Wentz et al. 2007; Chung et al. 2014). Monitoring of monthly TPW is available from satellites and reanalyses for the recent period (Kidder and Jones 2007; Li and Chen 2005; Sudradjat et al. 2005). Trenberth et al. (2005, 2011) found that over oceans the variability and trends in TPW from most dynamic reanalyses and satellite estimates seem questionable, although they believe that the Remote Sensing Systems (RSS) reprocessed oceanarea estimate of Wentz et al. (2007) is superior. For periods longer than the satellite-sampling period, an analysis of some type is necessary to infer TPW from historical observations. Dynamic reanalyses assimilate observed data into a dynamic atmospheric model and can be produced for an extended period depending on what historical data are assimilated (e.g., Compo et al. 2011). However, as noted above, there may be problems with reanalysis TPW estimates. In this study, we use the higher-quality RSS satellite estimates to evaluate historical TPW variations from a dynamic reanalysis and 
develop statistical improvements for those historical estimates.

Besides improving TPW from a dynamic reanalysis, this study also serves as a test to see in general how well reanalysis climate fields may be improved using statistical methods and a combination of modern and historical data. Among the considerations evaluated are the relative skills of different historical estimates at different time scales and how to best combine them to maximize skill.

The next section describes the data, including comparisons of the satellite and extended reanalysis TPW estimates for their overlap period. That is followed by a description of methods, including the reconstruction method, statistical properties of the satellite TPW anomalies, time filtering for evaluating multidecadal variations, and forming superensembles. Cross-validation methods and results are also described, followed by a discussion of analysis results. The final section gives a summary of results and conclusions from the study, including some discussion of how our results reflect climate variations over the period of record.

\section{TPW data}

The TPW data and historical estimates are discussed here. All datasets are interpolated or averaged to a monthly and $2^{\circ}$ spatial grid to match the monthly output grid used by the historical Twentieth Century Reanalysis. The $2^{\circ}$ grid is centered on $0^{\circ}-358^{\circ} \mathrm{E}$ by $90^{\circ} \mathrm{S}-$ $90^{\circ} \mathrm{N}$. Data are used only over ocean areas because the satellite-based analysis is limited to those regions.

\section{a. Dynamic Twentieth Century Reanalysis}

The Twentieth Century Reanalysis (R20C) (Compo et al. 2011) uses observed sea surface temperature (SST) as the lower boundary for an atmospheric dynamic model that assimilates observed sea level pressure (SLP) and surface pressure. The SST used is monthly while the pressures assimilated are synoptic. Such an approach can produce a range of spatially complete climate variations anchored to the observed SST and SLP variations. Since records of SST and SLP extend into the nineteenth century, very long analyses of climate variables can be produced.

For this study, the R20C version 2 historical TPW is the first historical estimate. That estimate is evaluated, and methods for improving it are developed using additional information and statistical analysis. Monthly R20C TPW is on a $2^{\circ}$ spatial grid for 1871-2012. The R20C data were obtained from NOAA/ESRL (http:// www.esrl.noaa.gov/psd/data/gridded/).

\section{b. Satellite-based RSS analysis}

The RSS analysis of oceanic monthly TPW is a satellitebased analysis available beginning January 1988 [see Remote Sensing Systems (2014) for details]. The RSS data were obtained on a monthly and $1^{\circ}$ spatial grid. Small gaps in the ocean-area analysis caused by islands, coastlines, and ice are filled using interpolation from surrounding regions. The filled analysis is then averaged to the monthly and $2^{\circ}$ spatial grid.

Overall, the R20C and RSS analyses yield similar results for the 1988-2012 overlap, but there are some important differences. Systematic differences are evident in the 1988-2012 climatologies of the two (Fig. 1). Although the large-scale spatial patterns are essentially the same, the R20C slightly underestimates values associated with tropical convergence and overestimates values in most other regions relative to RSS values. A $t$ test of the differences of the means indicates that they are not significant at the $95 \%$ level over most midlatitude regions. They are more commonly significant in the tropics, where values are high, and at high latitudes, where the variance is low. Besides the mean biases, there are also annual-cycle differences, as shown by the first empirical orthogonal function (EOF) of the monthly climatology difference (Fig. 2). The first difference mode accounts for almost two-thirds of the annual-cycle difference and shows that in the warm season the tropical R20C values tend to be too weak relative to RSS values. In both climatologies the warm-season tropics have larger TPW values, and this difference mode indicates that the R20C annual cycle tends to be too weak.

For both the R20C and RSS TPW, anomalies are formed using the separate 1982-2012 climatologies for each dataset. Here we perform anomaly analyses, which do not reflect the large climate differences discussed here. A simple first-order correction of the R20C analysis could be performed using a climate adjustment to remove these differences. Although anomaly errors are smaller than climatology errors, they can still influence climate studies.

\section{c. Statistical estimate from SST}

As discussed above, the R20C dynamically infers TPW from historical SST and SLP. Statistical historical estimates may also be made. Here we apply a method for estimating TPW from SST records that begin in the nineteenth century.

The monthly TPW can be estimated from SST because of the Clausius-Clapeyron relationship between saturation water vapor pressure and temperature. A relationship between SST and TPW was shown by Stephens (1990) for SST > $288 \mathrm{~K}\left(15^{\circ} \mathrm{C}\right)$ : 

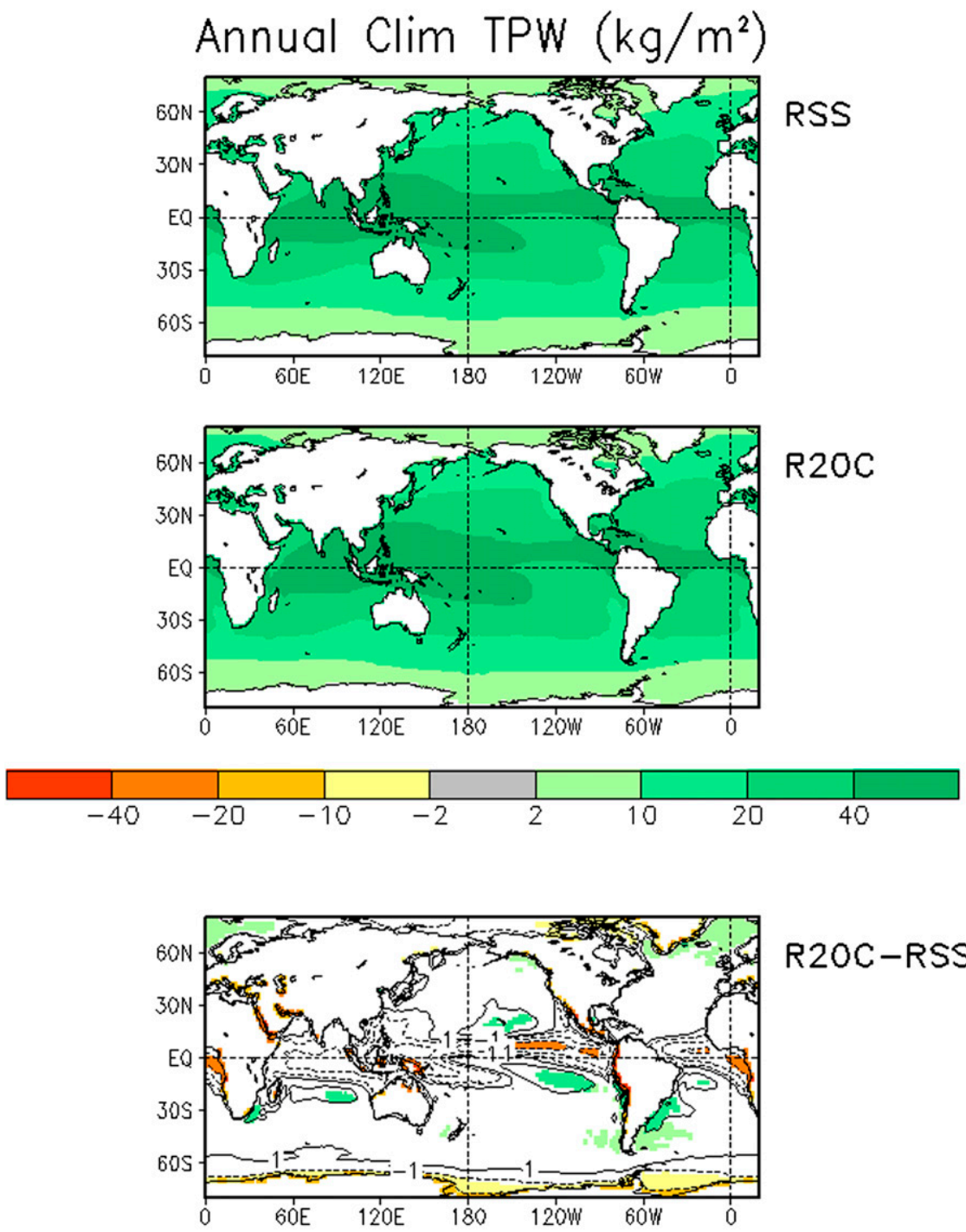

R20C-RSS

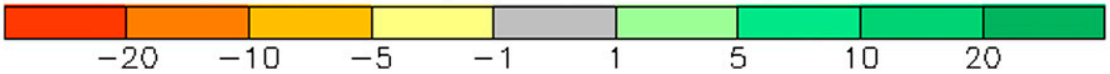

FIG. 1. (top) Annual mean 1988-2012 TPW climatology from RSS and R20C and (bottom) their difference $\left(\mathrm{kg} \mathrm{m}^{-2}\right)$. Differences are plotted with contours, with shading to indicate $95 \%$ significance.

$$
W(\mathrm{SST})=10.82\left(\frac{r}{1+\lambda}\right) e^{a\left(T_{k}-288\right)} .
$$

In Eq. (1), $W$ is the TPW estimate in centimeters and $T_{k}$ is the SST in kelvins. The constants were estimated by fitting Eq. (1) to data. Stephens (1990) found best fits using $a=0.0686$ and $[r /(1+\lambda)]=0.162$. For our evaluations, we use these published constants and multiply results by 10 to convert the units to kilograms per cubic meter.

To make historical estimates, we use the uninterpolated bias-adjusted SST data of Kennedy et al. (2011a,b). Bias adjustments are needed because of the changing mix of SST observing methods over the historical period. This dataset gives uninterpolated monthly SST anomalies on a $5^{\circ}$ spatial global grid beginning 1850 and updated periodically.

To make the SST climatology consistent with the relationship between $W$ and SST, we use the RSS 19882013 monthly TPW to estimate monthly SST from the inversion of Eq. (1):

$$
T_{k}=\frac{1}{a} \ln \left[\frac{W}{10.82\left(\frac{r}{1+\lambda}\right)}\right]+288 .
$$



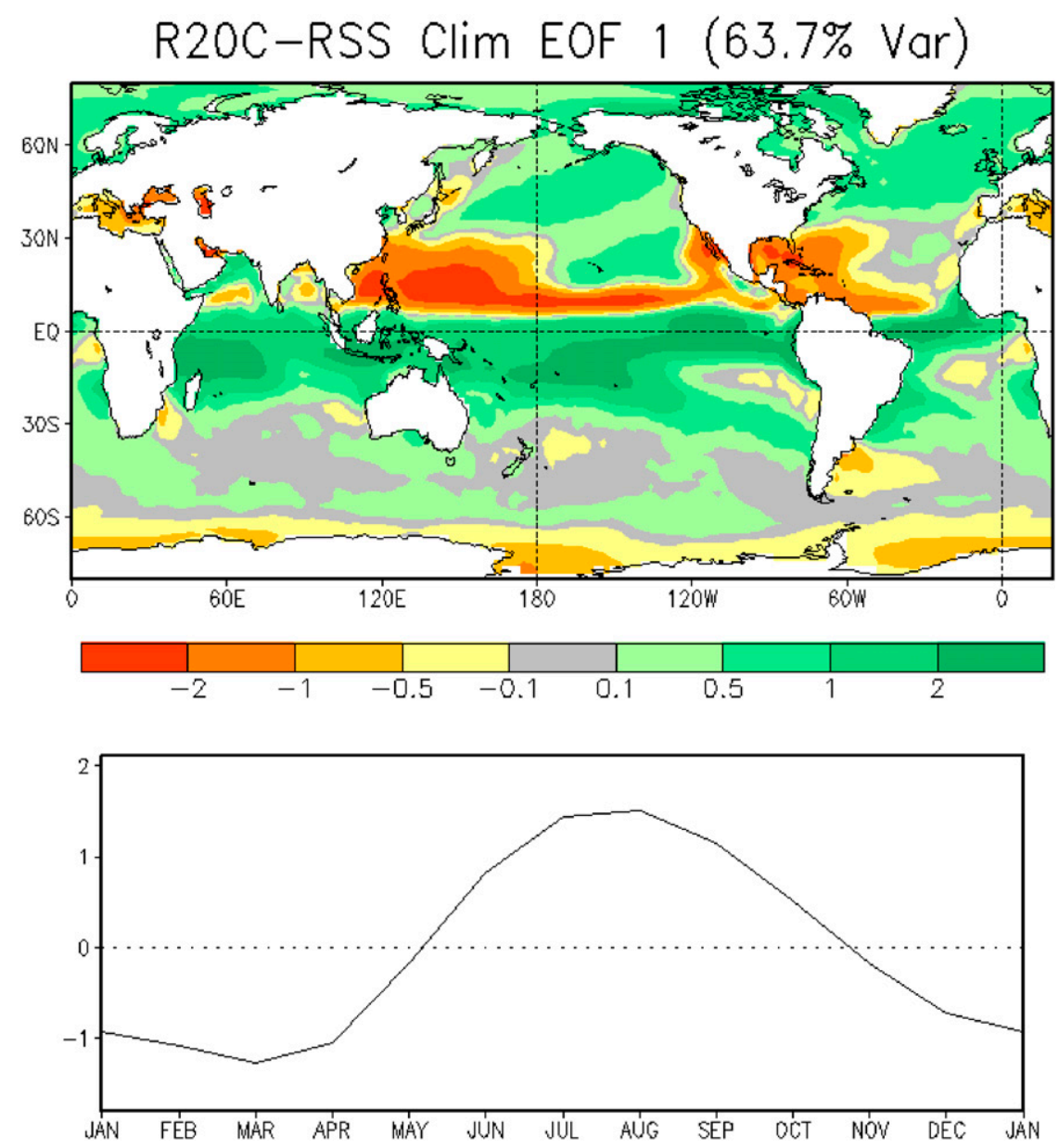

FIG. 2. First covariance-based EOF of the monthly 1988-2012 R20C - RSS climatology difference $\left(\mathrm{kg} \mathrm{m}^{-2}\right)$. (top) The spatial loadings of the EOF and (bottom) the EOF time series associated with the monthly climatology differences.

The resulting SST climatology is generally consistent with a directly computed SST climatology in its spatial and seasonal variations for SST warmer than about $10^{\circ} \mathrm{C}$. We recenter the SST anomalies to the climate base period and use them with this derived climatology to estimate the monthly SST, keeping all values $15^{\circ} \mathrm{C}$ or warmer. These monthly SSTs are used with Eq. (1) to estimate monthly TPW from 1850 to 2013 . We refer to these SST based estimates of TPW as WSST.

We do not expect the relatively simple SST-based estimate of TPW to be as good as the R20C estimates. Potential sources of errors in WSST include uncertainties in historical SST, uncertainties in Eq. (1) parameters, and the fact that TPW is influenced by factors other than surface temperature, such as water vapor advection. The R20C estimates should better resolve all the processes responsible for TPW variations. These uncertainties will all influence the error estimate of the reconstructed WSST discussed later. In addition, there is no WSST estimate in regions where $\mathrm{SST}<15^{\circ} \mathrm{C}$, so the reconstructed WSST is more dependent on spatial covariance to estimate values at high latitudes, which also tends to increase the reconstructed WSST error estimates. However, the WSST estimates and reconstructions based on them could be useful for helping to understand interactions between TPW and SST over the historical period. In addition they could contain independent information that may improve the historical estimates, so we use them for testing and evaluations.

\section{Statistical analysis methods}

This section describes statistical methods used for historical TPW anomaly reconstruction, cross-validation methods used to evaluate the anomaly reconstructions, and superensemble methods used to form improved reconstructions. Evaluations of reconstruction anomalies are relative to the RSS TPW anomalies. 


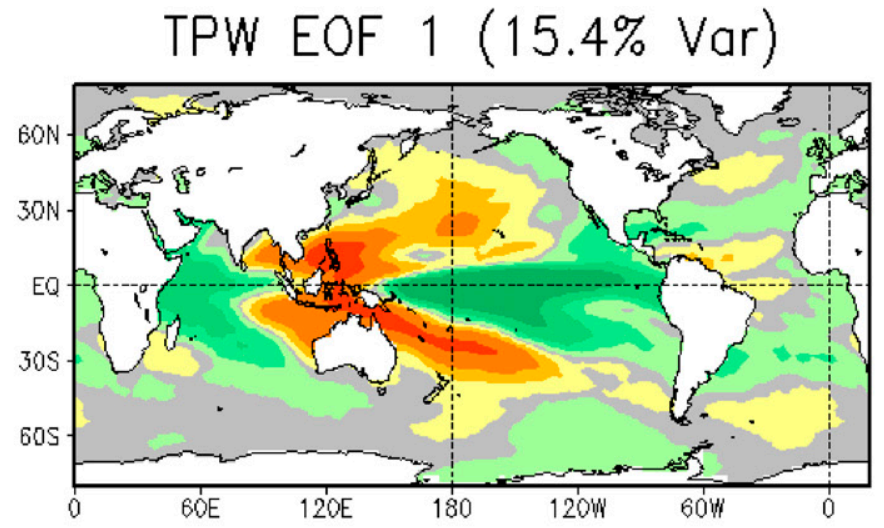

TPW EOF 2 ( $7.5 \%$ Var)
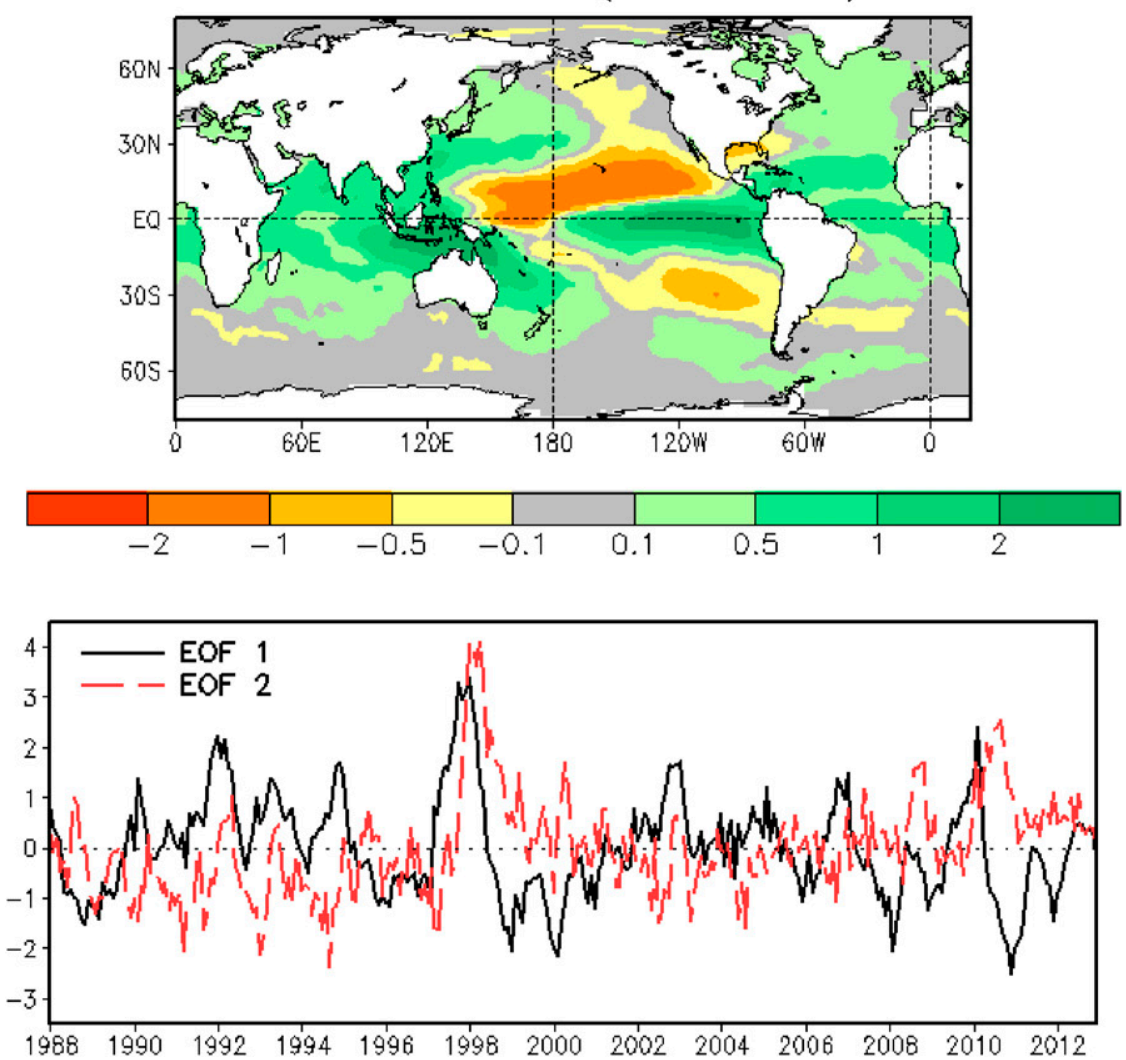

FIG. 3. First and second EOFs of monthly RSS TPW anomalies, 1988-2012, respectively. Spatial patterns are shown for (top) EOF 1 and (middle) EOF 2 and (bottom) their time series. Units of time series multiplied by spatial loading values are kilograms per meter squared.

\section{a. Statistical reconstructions}

The reconstruction method was developed for historical SST analysis (Smith et al. 1996) and is described in appendix A. It is a method for analyzing sparsely distributed data by using a set of spatial functions computed from a period with complete sampling. The available data anomalies are fit to the set of spatial functions to compute weights for each function. The weighted sum of the functions gives the reconstruction. Here we use spatial functions defined by covariance EOFs of the RSS TPW anomalies. Data fit to the spatial functions are anomalies from one of the historical TPW estimates.

The first two EOF modes are shown as example spatial functions (Fig. 3). The leading modes describe largescale variations that are strongest in the tropics. Both the 
patterns and their associated time series indicate that much of the variations represented are associated with El Niño-Southern Oscillation (ENSO). Correlations with the Climate Prediction Center (CPC) monthly OISST Niño-3.4 index (www.cpc.ncep.noaa.gov/data/ indices/) are 0.92 for mode 1 with 0 lag. For mode 2 correlation is highest with the index lagged 6 months, when it is 0.49 . Mode 2 partly represents a later stage of ENSO variation, although the weaker correlation indicates that much of its variance is not associated with ENSO. Modes are ordered so that as the mode number increases, the amount of base-period variance it accounts for decreases. So although the first two modes account for more than $20 \%$ of the variance, the first 10 account for only about $44 \%$. The first 50 modes account for about $76 \%$ of the base variance, the first 75 for about $84 \%$, and the first 100 for about $89 \%$. The higher modes each account for little base variance and tend to describe smallscale, short-period variations. For example, individual modes beyond the first 50 each account for $\leq 0.4 \%$ of base variance, beyond the first 75 for $<0.3 \%$, and beyond the first 100 for $<0.2 \%$ of base variance. A more complete discussion of EOF properties is given by Davis (1976), who discusses how EOFs can be used to perform analyses. In addition, a test for the significance of higher EOF modes was suggested by North et al. (1982). That test compares a first order estimate of sampling error to the difference between adjacent modes. When the variance difference reduces to the level of sampling error, the modes cannot be clearly distinguished. Using that test, the first 50 modes can be distinguished, but variance differences between higher modes are about the same or smaller than the sampling-error estimate. In another section, cross-validation tests are used to define the best set of modes for analysis. Since higher-mode variations could reflect errors, and because what signal they resolve may not be reliably resolved by the historical data, it is best to not use them in a reconstruction.

\section{b. Cross-validation reconstructions}

Cross-validation analysis is a way to simulate historical conditions in the modern period. Here the crossvalidation reconstructions are computed using either the R20C data or the WSST data. Spatial functions for the cross-validated reconstructions are computed using the RSS TPW anomalies that omit data from $3 \mathrm{yr}$ centered on the year of the cross-validation analysis month. This gives a set of spatial functions for each year that should be independent of variations within that year, simulating the use of base-period functions to reconstruct independent historical periods.

The R20C sampling is constant while the WSST sampling can change over the historical period. However, using the sampling test described by Smith et al. (1998) and a critical value of $5 \%$ variance sampled, the WSST sampling is adequate to sample most modes over the SST record period and all modes in the overlap analysis period. In addition, although the R20C output has no gaps, the input historical SST used for R20C is based on similarly sparsely sampled data, so there is some justification for using the modern sampling for both to give a fairer estimate of their relative skill. For these reasons we do not use historical WSST sampling masks in our crossvalidation analysis. As discussed later, even without historical sampling masks, the WSST reconstruction has much lower skill.

\section{c. Time filtering}

We consider both unfiltered and time-filtered results to help evaluate how well the analysis performs on different time scales. Here we filter annual averages using a 7-yr binomial filter to concentrate on multidecadal variations. The filter and how it influences the number of degrees of freedom is discussed in appendix B.

\section{d. Superensembles}

A superensemble is a weighted sum of individual estimates using weights that reflect the relative reliability of the individual estimates. If we use an optimum averaging approach for data at a point, then the individual weights can be computed from the relative error variance of each estimate:

$$
w_{i}=\frac{1}{1+\eta_{i}^{2}} .
$$

Here the subscript is used to indicate that there may be many different estimates, and $\eta_{i}^{2}$ is the noise-signal variance ratio for the estimate. In practice, we wish to normalize weights so that the ensemble variance will not be damped. For a set of $n$ estimates, the normalized weights may be computed as

$$
W_{i}=w_{i} / \sum_{j=1}^{n} w_{j} .
$$

To compute the noise-signal variance ratio for an estimate, first consider the true value of a variable $y$ and its estimate $x_{i}$. Assuming no offset between variables, which is appropriate for the anomalies considered in this study, we may express the variable as

$$
x_{i}=\alpha_{i} y+\varepsilon_{i} .
$$

Here $\alpha_{i}$ represents the error in the amplitude of the estimate, which we will assume to be constant. The random error $\varepsilon_{i}$ represents all other errors. The assumption of randomness is employed here because we wish to estimate 
noise. In reality, there could be correlated and bias errors not accounted for by amplitude, so this should be thought of as a first-order estimate of the noise to be used for weighting individual ensemble inputs. The weighting only uses the random error through the noise-signal ratio. Spatially or temporally correlated errors and biases do not influence the weights in this estimate, although the presence of those errors will influence the overall error of the ensemble. This method of computing the superensemble minimizes the error of the ensemble if the nonrandom errors in the individual members are comparable. However, if one member had a large bias but a low noise estimate, then the ensemble would strongly reflect that bias. A more complete discussion of errors in general is given by Kagan (1979).

Using these definitions, the variance of the estimate can be expressed as a function of the variance of $y, \sigma^{2}$, and the random-error variance $\left\langle\varepsilon_{i}^{2}\right\rangle$ :

$$
\sigma_{i}^{2}=\alpha_{i}^{2} \sigma^{2}+\left\langle\varepsilon_{i}^{2}\right\rangle
$$

Here the angle brackets indicate averaging. Typically, time averaging is used in practice. Similarly, the square of the correlation between the estimate and $y$ can be expressed as

$$
r_{i}^{2}=\frac{\alpha_{i}^{2} \sigma^{2}}{\alpha_{i}^{2} \sigma^{2}+\left\langle\varepsilon_{i}^{2}\right\rangle} .
$$

Note that $\alpha_{i}^{2} \sigma^{2}$ is the signal variance for the estimate. The random-error variance is the noise variance for the estimate. Thus, the estimate noise-signal error variance ratio may be written as

$$
\eta_{i}^{2}=\frac{1-r_{i}^{2}}{r_{i}^{2}} .
$$

Note that substituting Eq. (8) into Eq. (3) yields $w_{i}=r_{i}^{2}$, making the ensemble weights proportional to the variance explained by each member. The correlations between individual estimates and the true value of a variable can be computed by assuming that the RSS or a smoothed version of RSS approximates the truth. This allows us to compute approximately optimal weights for forming a superensemble. Crossvalidation correlations should be used so that the superensemble weights are valid for the historical period. Note that there could be common errors in the various TPW estimates from errors in the SST, which is used to help produce the ensemble members. However, this estimate of the weights does not consider error correlations between the individual ensemble members but only the random error estimate of each member.

The noise-signal error variance from Eq. (8) is used to find weights for the individual inputs, which in our case is the individual reconstruction anomalies. Since the same set of anomaly reconstruction modes are used for each input reconstruction, we could in principle form an ensemble using separate ensemble weights for each mode. Of course, that would require estimates of errors for individual modes. Here we use a simpler approach, using cross validation to estimate correlations for the full reconstruction of each ensemble input. Uncertainty in each individual reconstruction is from uncertainty in the EOF weight estimates for the set of modes, which is reflected in the cross-validation correlation. Note that all reconstructions are of anomalies from the RSS climatology, and we assume that there are no errors in that annual cycle.

We do not use the cross-validation results to estimate the error directly because the ensemble weights are based on the noise of each input, while the cross-validation error may contain a combination of noise, sampling, and bias errors. Using the cross-validation results to estimate correlations for computing weights ensures that we only consider random errors in the weighting, although in practice the cross-validation correlations and total rootmean-square errors (RMSEs) tend to be inversely proportional. Thus, using the total cross-validation RMSE would not greatly change the relative weighting of the ensemble members.

In this study, cross validation is used to estimate errors of each individual input. This has the advantage of allowing reasonable estimates to be computed for all inputs in a consistent way. However, there are other ways to estimate errors for the different inputs. For WSST, the error estimates for historical SSTs could be used to compute a range of possible WSST results, and those in turn could be reconstructed to estimate R[WSST] errors. Here, R[WSST] is the statistical reconstruction of TPW using WSST data. Since R20C is computed from an ensemble reanalysis, the spread among the individual ensemble members could be used to define an error estimate. Reconstruction of the individual R20C members would yield a range of R[R20C] that could be used to estimate its errors.

Reconstruction error for each input is computed here using the cross validation. The error of a weighted average can be found using error characteristics of the individual members (Kagan 1979; Smith et al. 1994). Here the error estimate for the average is simplified since all ensemble members are always present, so there is no ensemble-sampling error, reducing the ensembleaverage error to 


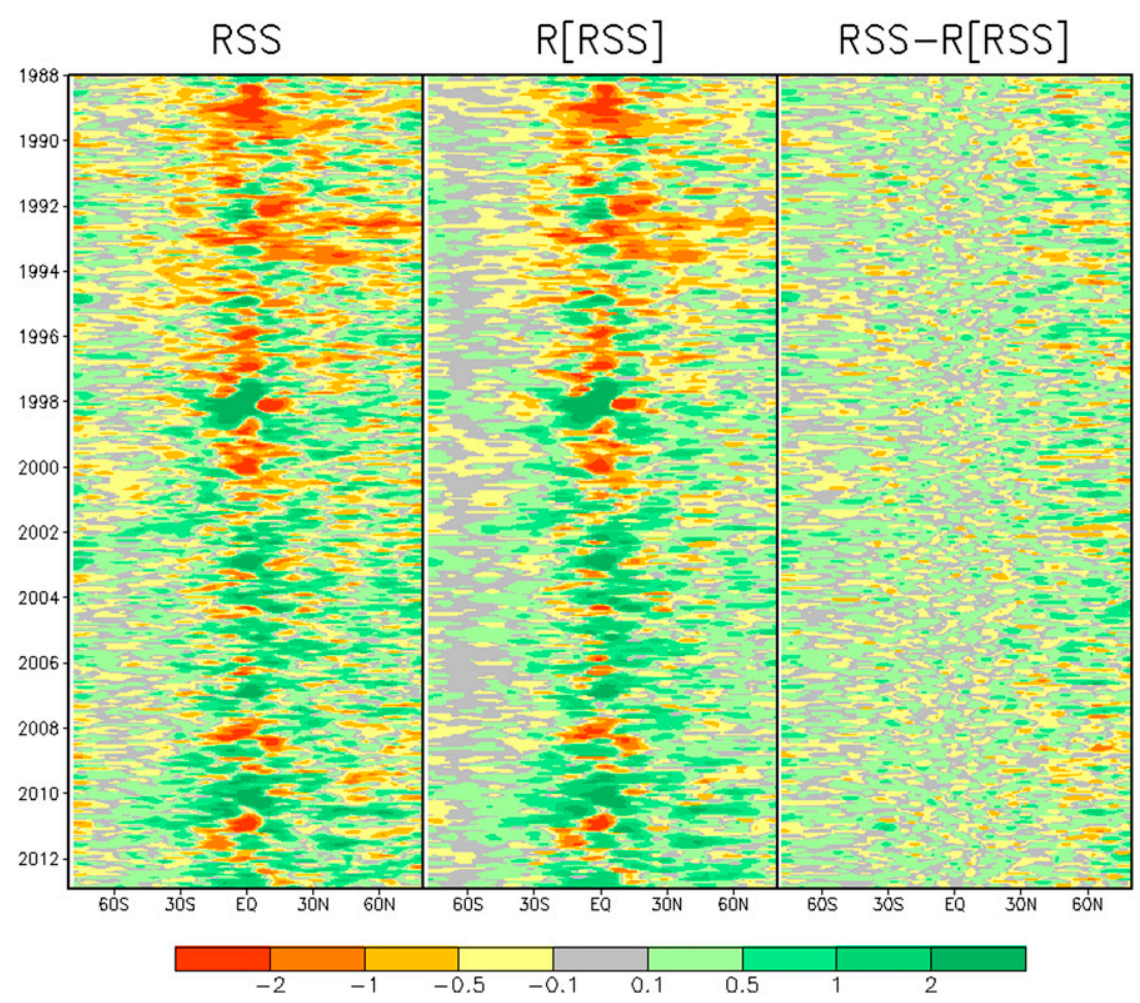

FIG. 4. Zonal averages of monthly TPW anomalies from (left)-(right) RSS, 50-mode smoothed $\mathrm{R}$ [RSS], and their difference $\left(\mathrm{kg} \mathrm{m}^{-2}\right)$.

$$
E_{A}^{2}=\sum_{i=1}^{n} W_{i}^{2} \varepsilon_{i}^{2}
$$

Here $\varepsilon_{i}^{2}$ is the error for the member. For normalized error we can use the noise-signal variance ratio estimate instead of $\varepsilon_{i}^{2}$. The full error can be estimated by multiplying the normalized error by the ensemble variance.

\section{Cross-validation results}

Cross-validation testing, described earlier, is applied to show the skill that can be expected from different reconstructions. Skill is computed relative to the RSS anomalies. Both the reconstruction skill and the R20C skill are compared to show skill improvements for both monthly and multidecadal filtered reconstructions.

\section{a. Monthly results}

Before evaluating cross-validation tests, it is useful to consider what the tests should be validated against. One possible validation dataset is the RSS anomalies. Another is the RSS anomalies smoothed by reconstruction, here referred to as R[RSS]. Since R[RSS] is a dependentperiod reconstruction, its anomalies should represent all of the large-scale variations most important for climate studies. This is demonstrated by comparing zonal means of RSS and R[RSS] using a 50-mode dependent-period reconstruction (Fig. 4). Both zonal means indicate essentially the same climate variations, including both ENSO and multidecadal changes. Differences tend to be small and noisy. As we discussed earlier, additions to baseperiod modes higher than mode 50 may be strongly influenced by sampling error, so higher modes should be used with caution. The cross-validation testing helps to better define what skill additions may be expected from higher modes. We will validate against both RSS and $\mathrm{R}$ [RSS] anomalies and discuss how to use the results in developing a reconstruction.

The monthly cross-validation tests are performed using 50, 75, and 100 EOF functions for analysis. Validation is against RSS anomalies and R[RSS] anomalies for 1988-2012. The R[RSS] validation data use the same number of EOF functions as the cross validation to simulate similar smoothing. Note that although the number is the same, they are different sets of EOFs since the crossvalidation EOFs are independent.

Overall skill of the different analyses is evaluated using the global averages of temporal correlation (see Table 1). For the monthly results the correlation with RSS is highest for the R20C output, while the correlations for the others increase with more EOF modes. That shows that much of the correlation skill is associated 
TABLE 1. Monthly cross-validation correlations (corr) with RSS and R[RSS] using the indicated number of cross-validated modes. Cross-validation reconstructions are $\mathrm{R}[\mathrm{R} 20 \mathrm{C}]$ and $\mathrm{R}$ [WSST]. The unaltered R20C analysis is also validated for comparison. Shown are global averages of correlations computed at each location. For both RSS and R[RSS], the highest correlations for each analysis are in boldface.

\begin{tabular}{clcc}
\hline \hline Modes & Analysis & $\begin{array}{l}\text { RSS } \\
\text { corr }\end{array}$ & $\begin{array}{c}\text { R[RSS] } \\
\text { corr }\end{array}$ \\
\hline \multirow{2}{*}{50} & R[WSST] & 0.298 & $\mathbf{0 . 4 2 4}$ \\
& R[R20C] & 0.528 & $\mathbf{0 . 7 6 9}$ \\
& R20C & 0.753 & 0.630 \\
75 & R[WSST] & 0.311 & 0.396 \\
& R[R20C] & 0.581 & 0.760 \\
100 & R20C & 0.753 & 0.680 \\
& R[WSST] & $\mathbf{0 . 3 1 7}$ & 0.376 \\
& R[R20C] & $\mathbf{0 . 6 1 2}$ & 0.748 \\
& R20C & 0.753 & $\mathbf{0 . 7 0 9}$ \\
\hline
\end{tabular}

with the small-scale variations discussed above. Validation against $\mathrm{R}[\mathrm{RSS}]$ is best for the reconstructed R20C $(\mathrm{R}[\mathrm{R} 20 \mathrm{C}])$ using 50 modes. The cross-validation reconstruction using WSST (R[WSST]) has the lowest skill, but measured against R[RSS] its skill is best when 50 modes are used.

For the 25-yr cross-validation record, individual monthly correlations above 0.11 are significant at the 95\% level. Here the correlation significance method uses an $F$ test and is described in Panofsky and Brier (1958). Clearly, the average monthly correlations in Table 1 are significant, as are the correlations for almost all regions (Fig. 5).

Using Eq. (8), these correlation values can be used to estimate overall normalized error of each estimate. From the 50 mode values validated against R[RSS], the standard error of R[WSST] is about 6.6 times larger than the $\mathrm{R}[\mathrm{R} 20 \mathrm{C}]$ standard error. Using the same validation, the $\mathrm{R} 20 \mathrm{C}$ standard error is 1.5 times larger than the $\mathrm{R}[\mathrm{R} 20 \mathrm{C}]$ standard error. The relatively large R[WSST] error is consistent with Stephens (1990), who found relatively large error estimates associated with the WSST method. Here we do not use the Stephens (1990) error estimates, but instead use the monthly global crossvalidation error estimates validated against R[RSS] to form the superensemble estimate. We estimate the noisesignal error variance from Eq. (8) and then compute the normalized weights using Eqs. (3) and (4). Because the $\mathrm{R}$ [WSST] error variance is so much larger than the others, it contributes little to the ensemble estimate. However, we show later that including it does slightly improve the ensemble as shown by cross-validation testing.

For the analysis period considered here, regions with SST $>15^{\circ} \mathrm{C}$ produce enough WSST sampling to allow reconstruction using all modes for the overlap reconstruction period (beginning in 1871). Therefore, using a historical sampling grid for the cross-validation tests would have little influence on R[WSST] sampling error simulated by cross validation. There could be other SST errors not accounted for by cross validation and that contribute to R[WSST] uncertainty. In particular, SST bias uncertainty before the 1940s is not considered in the cross-validation error estimate. Including that error component would make the $\mathrm{R}$ [WSST] weights lower in the ensemble. However, those weights are already much lower than the weights for either R20C or R[R20C], so they contribute relatively little to the ensemble, and accounting for more error would not noticeably change the ensemble for the first half of the twentieth century.

Note that the relative errors used to weight ensemble members do not consider the spatial variations of errors, but only the global mean. Global errors are considered to give the ensemble with the best overall skill. There are spatial variations in the skill of each individual input. Validated against the R[RSS] all indicate most skill in the tropical Pacific, associated with ENSO variations (Fig. 5). In general, all inputs are best in the tropics, with lower skill in the extratropics. Because of the similarity of the spatial correlation patterns, in our ensemble we use only the global-average skill and only compute global ensemble weights. The greater drop off in skill with latitude for R[WSST], especially in the Southern Hemisphere, suggests that considering spatially variable weights could slightly improve the ensemble by giving that input more weight in the tropical Pacific and less in most other regions.

Using global weights, the ensemble weights for $\mathrm{R}$ [WSST], R[R20C], and R20C are roughly 0.15, 0.50, and 0.35 , respectively. With these weights the R[WSST] contribution is modest everywhere. The R[WSST] correlations are on average roughly 0.6 in the tropics and 0.3 near $60^{\circ}$ latitude. For R[R20C] the tropical and $60^{\circ}$ latitude average correlations are roughly 0.8 and 0.7 , while for R20C they are roughly 0.7 and 0.5 . Using these estimates, we can evaluate the approximate change in ensemble weighting if we were to use local weights. Local weights in the tropics for the three would be about $0.24,0.43$, and 0.33 , slightly increasing the influence of R[WSST] mostly at the expense of R[R20C]. Around $60^{\circ}$ latitude local weights for the three would be about $0.11,0.59$, and 0.30 , slightly reducing the influence of R[WSST] and giving more to R[R20C]. Thus, even large differences in correlations have a relatively modest influence on the ensemble weights because of the nonlinear relationship between the two. In the future, we may wish to use spatially varying ensemble weights, but the analysis done here suggests that it will have a modest influence on the ensemble. 

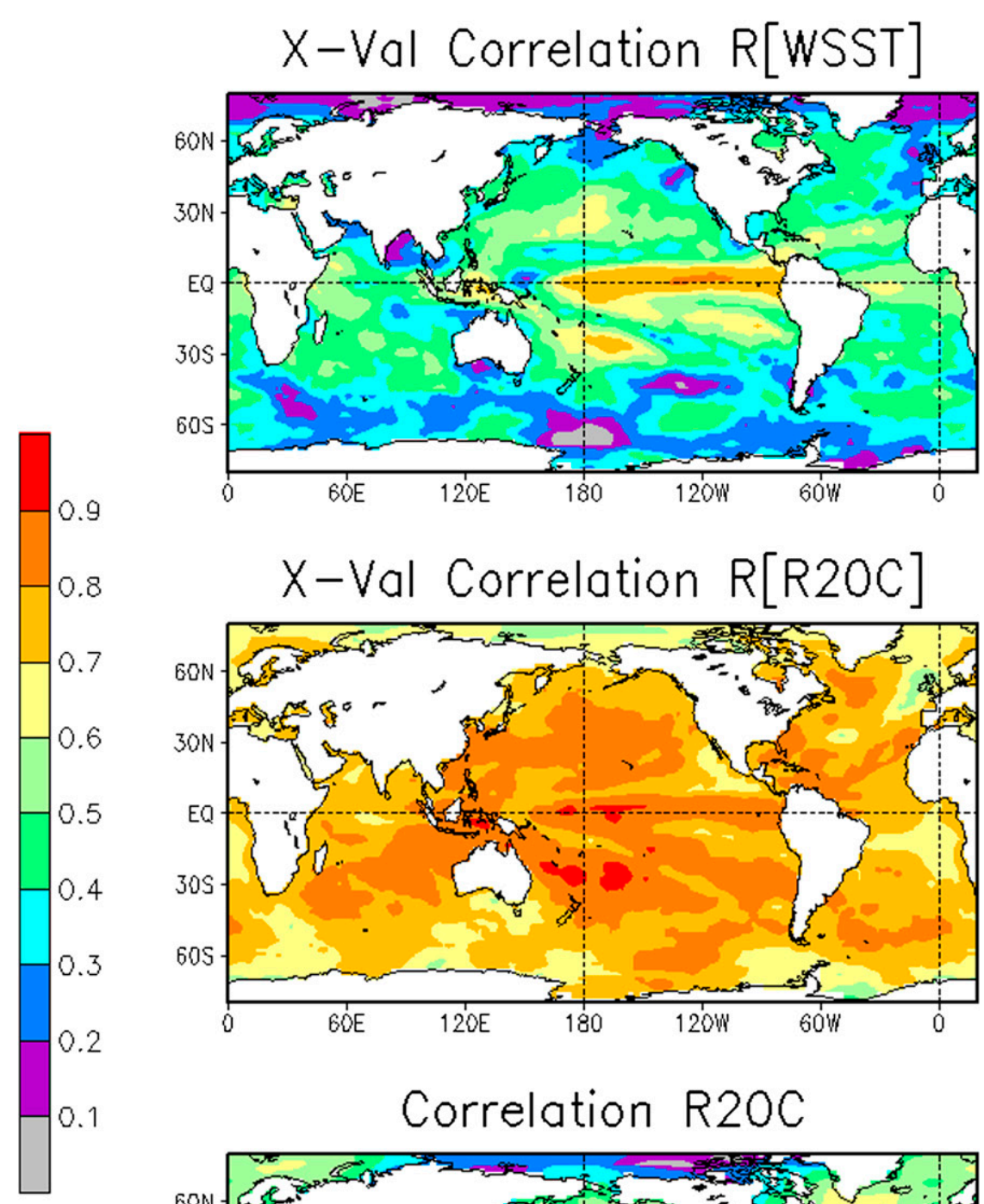

Correlation R20C

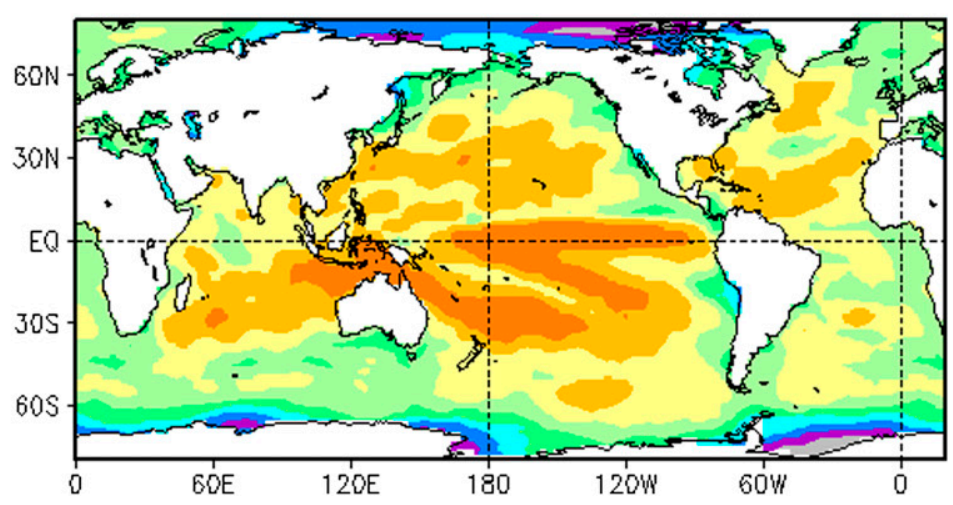

FIG. 5. Monthly cross-validation correlations with R[RSS] using 50 modes for (top) R[WSST] and (middle) R[R20C]. (bottom) The correlation of the unaltered R20C for the same period.

If we could be confident that the skill of the R20C anomalies did not change over time, then we could conclude that it would be the best analysis. However, the sampling of its input SST and SLP is lower in the historical period, especially before 1950 , as shown by sampling maps in Smith et al. (1994). Thus, many of the small-scale short-period features that lead to its higher correlation may be less reliable in the historical period. An examination of R20C ensemble spreads for the first and second half of the twentieth century indicates slightly larger spreads for monthly averages in the first half of the century, especially in regions that were 


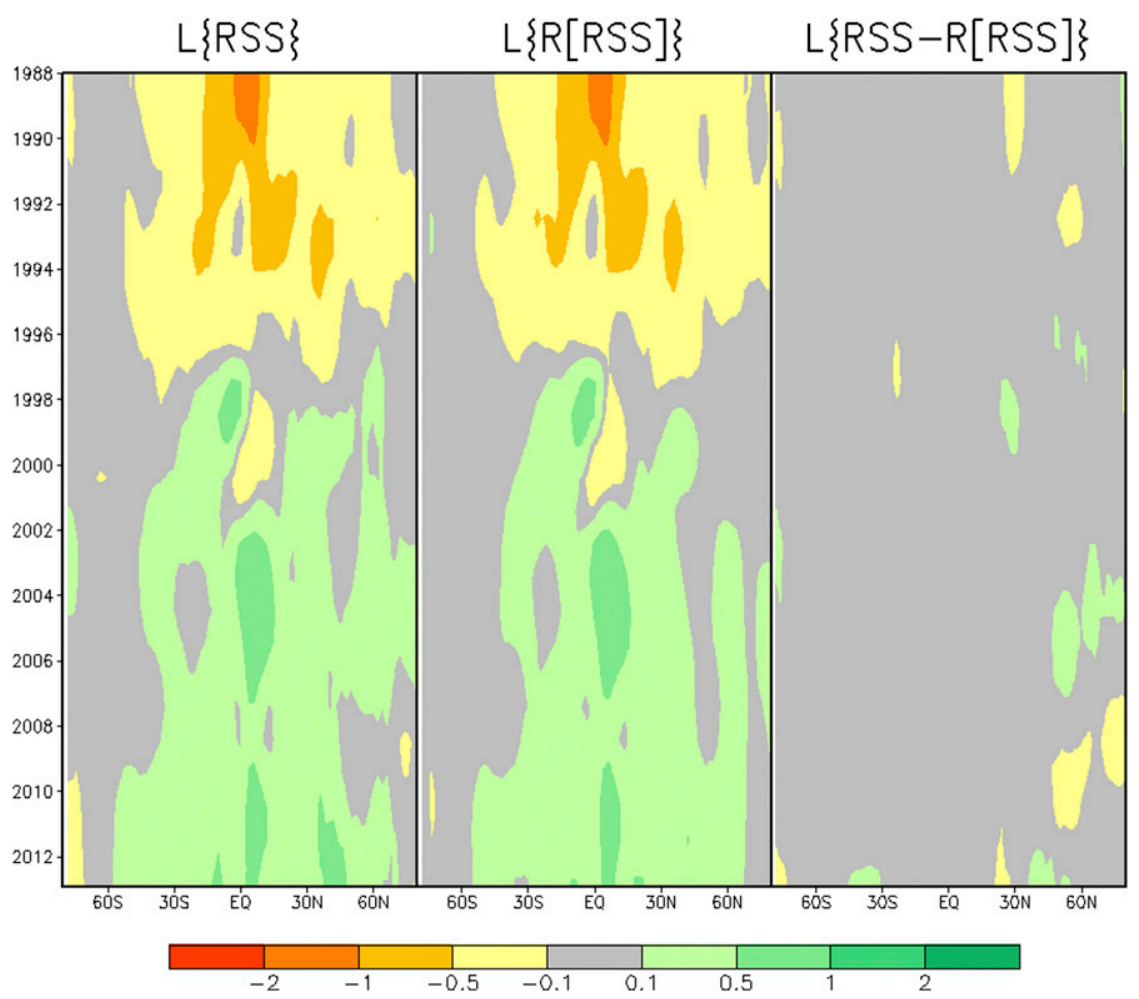

FIG. 6. Low-pass smoothed zonal averages of monthly TPW anomalies from (left)-(right) RSS, 50-mode smoothed R[RSS], and their difference $\left(\mathrm{kg} \mathrm{m}^{-2}\right)$.

sparsely sampled in that period. Those evaluations support the hypothesis that the R20C small-scale features are likely less reliable early in its record. For much of the historical period there may not be enough observations for R20C to reliably resolve such small-scale features, and some smoothing seems to be justified to provide more homogeneous variance resolution over the entire period of record. For these reasons, the R[RSS] validations may be a better indication of the relative historical skill.

\section{b. Low-frequency results}

It is useful to consider the cross-validation skill of the low-pass filtered TPW anomalies because these variations are critical for assessing multidecadal climate variations. Here the 7-yr binomial filter is used to define low-pass anomalies. Compared to the monthly zonal averages, the low-pass anomalies from RSS and 50-mode smoothed R[RSS] are nearly identical for the base period (Fig. 6). Thus, when considering climate variations, the small-scale and short-period monthly differences may be thought of as noise. It is possible that changes in the frequency of small-scale short-period variations could be related to climate. However, the monthly historical data used to estimate historical TPW may not be able to accurately simulate changes on those scales.
Conclusions from using low-pass filtered data are generally similar to conclusions from monthly data. Having more modes improves correlations against RSS, validation against $\mathrm{R}[\mathrm{RSS}]$ gives higher correlations for the reconstructions, and relative to $\mathrm{R}[\mathrm{RSS}]$ correlation is best using 50 modes. A difference is that for filtered data the R[WSST] correlation is much higher relative to the others. For example, with unfiltered data and validation against R[RSS], the R[WSST] correlation is $55 \%$ of the R[R20C] correlation (see Table 1), while for the comparable comparison using time-filtered data, R[WSST] correlation is $86 \% \mathrm{R}[\mathrm{R} 20 \mathrm{C}]$ correlation (see Table 2). That shows a close relationship between multidecadal SST and TPW variations, supporting the theoretical idea expressed by Eq. (1) that climatological changes in TPW are forced by temperature changes.

The decrease in the number of degrees of freedom caused by filtering is estimated using the method outlined in appendix B. For the cross-validation annual averages, we find that there tends to be little loss from the filtering done here, with R[WSST] showing the greatest loss, having a reduction factor of 0.84 . Since the method applied here gives only a crude estimate of the reduction factor, we use a factor of 0.8 for all crossvalidation annual values to be conservative in our confidence estimates. Applying that factor and testing for 
TABLE 2. Annual 7-yr smoothed cross-validation correlations (corr) with RSS and R[RSS] using the indicated number of crossvalidated modes. Cross-validation reconstructions are $\mathrm{R}[\mathrm{R} 20 \mathrm{C}]$ and $\mathrm{R}$ [WSST]. The unaltered R20C analysis is also validated for comparisons. Shown are global averages of correlations computed at each location. For both RSS and R[RSS] the highest correlations for each analysis are in boldface.

\begin{tabular}{clcc}
\hline \hline Modes & Analysis & $\begin{array}{l}\text { RSS } \\
\text { corr }\end{array}$ & $\begin{array}{c}\text { R[RSS] } \\
\text { corr }\end{array}$ \\
\hline \multirow{2}{*}{50} & R[WSST] & 0.497 & $\mathbf{0 . 6 2 4}$ \\
& R[R20C] & 0.584 & $\mathbf{0 . 7 2 7}$ \\
75 & R20C & 0.735 & 0.690 \\
& R[WSST] & 0.534 & 0.613 \\
& R[R20C] & 0.622 & 0.714 \\
100 & R20C & 0.735 & 0.721 \\
& R[WSST] & $\mathbf{0 . 5 4 4}$ & 0.599 \\
& R[R20C] & $\mathbf{0 . 6 3 5}$ & 0.700 \\
& R20C & 0.735 & $\mathbf{0 . 7 2 7}$ \\
\hline
\end{tabular}

significance indicates that filtered data with correlations of 0.44 are significant with $95 \%$ confidence. As with the monthly averages of correlations, the averages for filtered data are all significant.

These comparisons show that the analysis using 50 EOFs is best for reconstruction of climate variations through the historical period. Since we are most interested in such large-scale variations, we will use that number of modes for our reconstructions.

\section{c. Superensemble results}

Cross-validation ensembles of the analyses are next evaluated. Here ensembles are formed using the monthly cross-validation global correlations from Table 1 for the reconstructions using 50 EOFs. Methods described in section $3 \mathrm{~d}$ are used to compute normalized ensemble weights. Both monthly and low-pass filtered data are validated against both RSS and R[RSS]. Different ensembles of two or all three analyses are tested (Table 3). Ensembles of R[R20C] and R20C always yield higher correlations than R20C, showing that R20C is improved by combining it with a statistical analysis. Validated against R[RSS] there are further slight improvements from including the R[WSST] in the ensemble. The $\mathrm{R}$ [WSST] improvement is clearest in the low-pass-filtered ensemble correlations against low-pass-filtered R[RSS]. Without that smoothing, smaller-scale variations wash out the improvements.

The significance of an increase in correlation when adding an additional input to the ensemble can be tested using the same sort of test used for screening regressions, which typically add predictors until the explained variance does not increase significantly. For that situation an appropriate $F$-test value can be computed from the correlations with $p$ and $p-1$ predictors (e.g., Glahn
TABLE 3. Monthly (mo) and annual 7-yr smoothed (LF) crossvalidation correlations with RSS and R[RSS] using 50 cross-validated modes and global correlations to define weights. Cross-validation analyses used are R[WSST] (A), R[R20C] (B), and R20C (C). Either two or all three are merged as indicated. For each column the highest correlations are in boldface.

\begin{tabular}{lccccc}
\hline \hline & \multicolumn{2}{c}{ RSS } & & \multicolumn{2}{c}{ R[RSS] } \\
\cline { 2 - 3 } \cline { 6 - 6 } Ensemble & Mo corr & LF corr & & Mo corr & LF corr \\
\hline B,C & 0.758 & 0.746 & & 0.756 & 0.773 \\
A,B,C & 0.759 & 0.749 & & $\mathbf{0 . 7 7 0}$ & $\mathbf{0 . 7 9 4}$ \\
A,C & $\mathbf{0 . 7 6 1}$ & $\mathbf{0 . 7 5 6}$ & & 0.669 & 0.759 \\
A,B & 0.524 & 0.592 & & 0.763 & 0.742 \\
\hline
\end{tabular}

$1985)$ and is $\left(r_{p}^{2}-r_{p-1}^{2}\right)(n-p-1) /\left(1-r_{p}^{2}\right)$. For the monthly correlations validated against R[RSS] in Table 3, the change in mean correlation from test B,C $(r=0.756)$ to test $\mathrm{A}, \mathrm{B}, \mathrm{C}(r=0.770)$ is highly significant. As in our earlier discussions of correlations, here we use global average values as an estimate of typical values. This shows that in general it is better to include all three inputs in the ensemble. However, as discussed earlier, there is much spatial variation in the R[WSST] cross-validation correlation, and it is possible that including it in some regions while excluding it in others could improve the overall skill of the ensemble. Such testing could be included for the development of an improved analysis.

Comparing the global-average cross-validation correlation from $\mathrm{R}[\mathrm{R} 20 \mathrm{C}]$ alone relative to R[RSS] ( $r=$ $0.769)$ to the ensemble global-average correlation $(r=$ 0.770) using the same test indicates that although the ensemble correlations are typically higher, this slight improvement is not significant. For correlations of this magnitude, the monthly correlation needs to improve by roughly 0.005 to be significant with $95 \%$ confidence. Thus, considering only average skill, either R[R20C] or the ensemble could be used. In future studies, we hope to examine the ensemble method in more detail, including using spatially varying weights and developing regional cutoffs for excluding inputs with locally low skill. For the remainder of this study, we will evaluate the present ensemble with the understanding that improvements are possible.

These cross-validation analyses show that ensembles including R20C and statistical TPW analyses can improve the historical analysis. However, what ensemble should be used depends on what the ensemble is intended for. If a smooth climate analysis is desired for detecting and analyzing seasonal to multidecadal variations, then the best ensemble may be one that includes all three inputs and uses correlations relative to R[RSS] to compute ensemble weights. Improvements from including R[WSST] are clear for the time-filtered cross-validation analysis 
TABLE 4. Percent variance accounted for by each of the first 10 EOFs of annual superensemble reconstructed TPW anomalies, 1871-2012.

\begin{tabular}{ccccccccccc}
\hline \hline EOF & 1 & 2 & 3 & 4 & 5 & 6 & 7 & 8 & 9 & 10 \\
\hline Variance (\%) & 39.0 & 22.6 & 5.0 & 3.9 & 3.6 & 2.6 & 2.0 & 1.9 & 1.8 & 1.5 \\
\hline
\end{tabular}

validated against R[RSS]. That is how we weight individual analyses in the superensemble discussed below. Because of lower sampling and the relatively simple way of computing WSST, the R[WSST] may contain errors at smaller scales, and including it could reduce the resolution of some features, although its inclusion has little influence on the global-average correlations when validated against RSS. Thus, the requirements an analysis must meet should be considered when developing an ensemble analysis.

\section{Improved historical analysis}

The historical R[WSST] and R[R20C] 50 EOF reconstructions are computed for the R20C period (18712012) and a superensemble of all three is formed using monthly correlations relative to R[RSS]. Here we evaluate the results of the ensemble and compare the ensemble to individual analyses. Since we are most interested in climate variations, we consider annual averages. This is intended to demonstrate some of the potential value of the improved analysis, as defined by the cross-validation testing. More detailed analyses such as seasonal and monthly analyses are beyond the scope of this study.

\section{a. TPW annual EOFs}

First, consider the leading EOFs of annual-average anomalies from the superensemble. The first two EOFs account for over $60 \%$ of the variance and dominate the annual variations (Table 4). Modes 1 and 2 are dominated by multidecadal variations and ENSO variations, respectively (Fig. 7).

The spectrum of those time series is estimated using a Fourier decomposition to compute power as a function of frequency. The power estimates are normalized by the variance of the time series and slightly smoothed using a 1-2-1 filter, as suggested by Panofsky and Brier (1958). The estimates are then interpolated to regular period intervals for display (Fig. 8). Because of its trend, the first mode's energy increases with period, but it also indicates a peak at $10 \mathrm{yr}$ and a broad peak centered on $17.5 \mathrm{yr}$. The second mode has peaks at 6 and $13 \mathrm{yr}$ with smaller peaks at 9 and $21 \mathrm{yr}$. There is a suggestion for a peak at $71 \mathrm{yr}$ for the second mode, but the 142-yr record is not long enough to reliably resolve variations with that period. The multidecadal period peaks in the first mode may not be strongly associated with the Pacific decadal oscillation (PDO), which typically has 20-30-yr periods (Mantua et al. 1997; Biondi et al. 2001). However, the second mode suggests that PDO may be associated with modulating the strength of ENSO episodes. The trend-like change in the first mode can influence the character of ENSO episodes over long periods. The trends in the first two modes since the 1980s also suggest a changing character of ENSO episodes relative to earlier decades.

The first mode of the annual-average superensemble represents multidecadal variations, with some interannual variations superimposed. Interannual mode 1 variations are clearer in the detrended mode also shown in Fig. 7. Detrending is done by fitting a line to the time series that minimizes the mean-squared error of the fit and then subtracting that line from the time series. The interannual variations are often in phase with the second mode interannual variations, but they tend to have longer periods, as indicated by the spectral analysis. Before 1910 there is little multidecadal change and perhaps a slight negative trend, followed by a positive trend through the early 1940s. There is then little trend until after the 1970s, when a strong positive trend develops. This global multidecadal time series mirrors multidecadal global SST changes (e.g., Xue et al. 2003), showing the dependence of long-term TPW on surface temperatures. The first mode spatial patterns suggest an intensification of tropical TPW, although in the Pacific the increase is south of the typical intertropical convergence zone (Fig. 7, top). The only weak negative values on the map may be associated with atmospheric subsidence regions in the eastern Pacific. The patterns also indicate multidecadal increases over oceans adjacent to most monsoon regions, suggesting the potential for intensified monsoon rains. Monsoon changes generally consistent with these results have been shown by Zhang and Zhou (2011), Liu et al. (2012), and Lin et al. (2014).

Much stronger interannual variations are evident in the second mode of annual averages. That mode's spatial patterns (Fig. 7, middle) are positive in the eastern equatorial Pacific and negative in a horseshoe pattern in the western Pacific, typical of ENSO. While this mode has strong interannual variations, it has almost no trend until after the 1970s, when the time series develops a downward trend. That suggests a long-term decrease in eastern equatorial Pacific TPW associated with this mode in the most recent years. The first two modes indicate opposite changes in the eastern equatorial Pacific from different processes, suggesting that there may be a muted response in the total change in that region in recent years. That result is consistent with Dong and Zhou (2014) for SST changes. However, the frequency of variations in the 


\section{Super-Ensemble EOF 1 (39.0\%)}

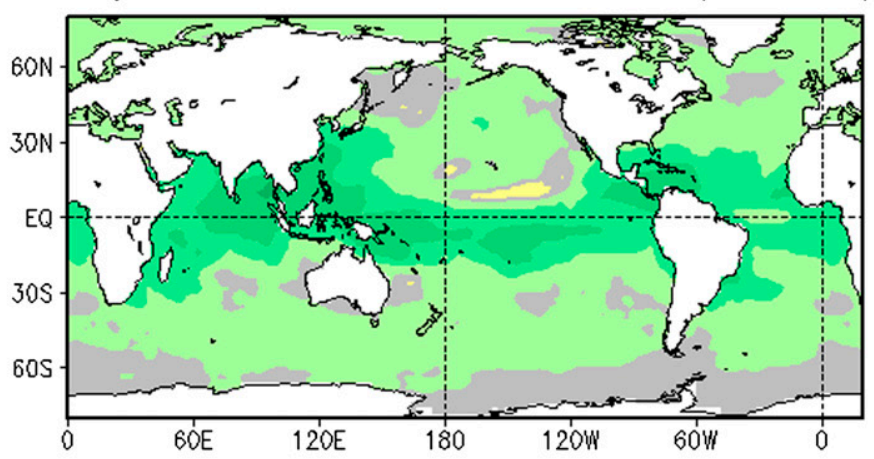

\section{Super-Ensemble EOF $2(22.6 \%)$}

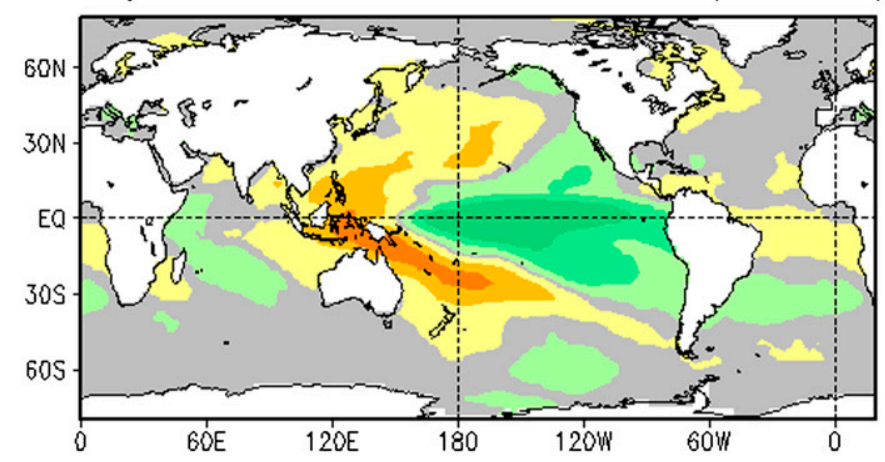

\begin{tabular}{|l|l|ll|l|l|l|l|l|l|}
\hline & & & & & & & & & \\
\hline-2 & -1 & -0.5 & -0.1 & 0.1 & 0.5 & 1 & 2
\end{tabular}

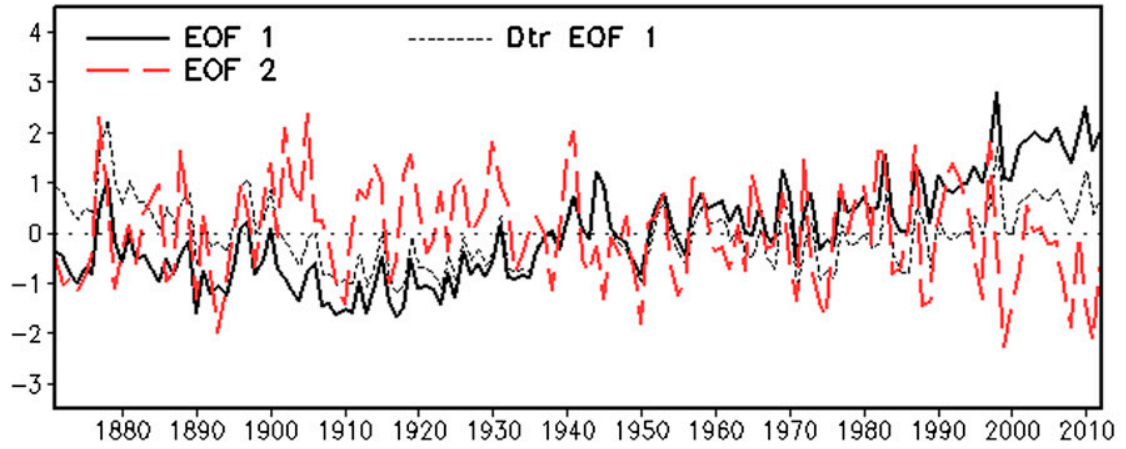

FIG. 7. First two covariance-based EOFs of annual average superensemble reconstructed TPW anomalies, 1871-2012. Spatial patterns are shown for (top) EOF 1 and (middle) EOF 2 and (bottom) their time series. Units of time series multiplied by spatial loading values are kilograms per meter squared. For comparison a linearly detrended version of the mode 1 time series is also shown (Dtr EOF 1).

region appears to have changed since the mid-1970s. Over the western Indian Ocean, there are similar counter trends in the first two modes. In the western Pacific, eastern Indian, and tropical Atlantic Ocean regions, the second mode suggests that after the 1970s, there is an intensification of the TPW increase associated with the first mode.
The first two modes account for over $60 \%$ of the annual variance, which should dominate the long-period changes. However, the recent opposite tendencies in the two modes can make it difficult to evaluate the overall tendency in that period. The trend in annual values over 1973-2012 was computed to evaluate the overall 


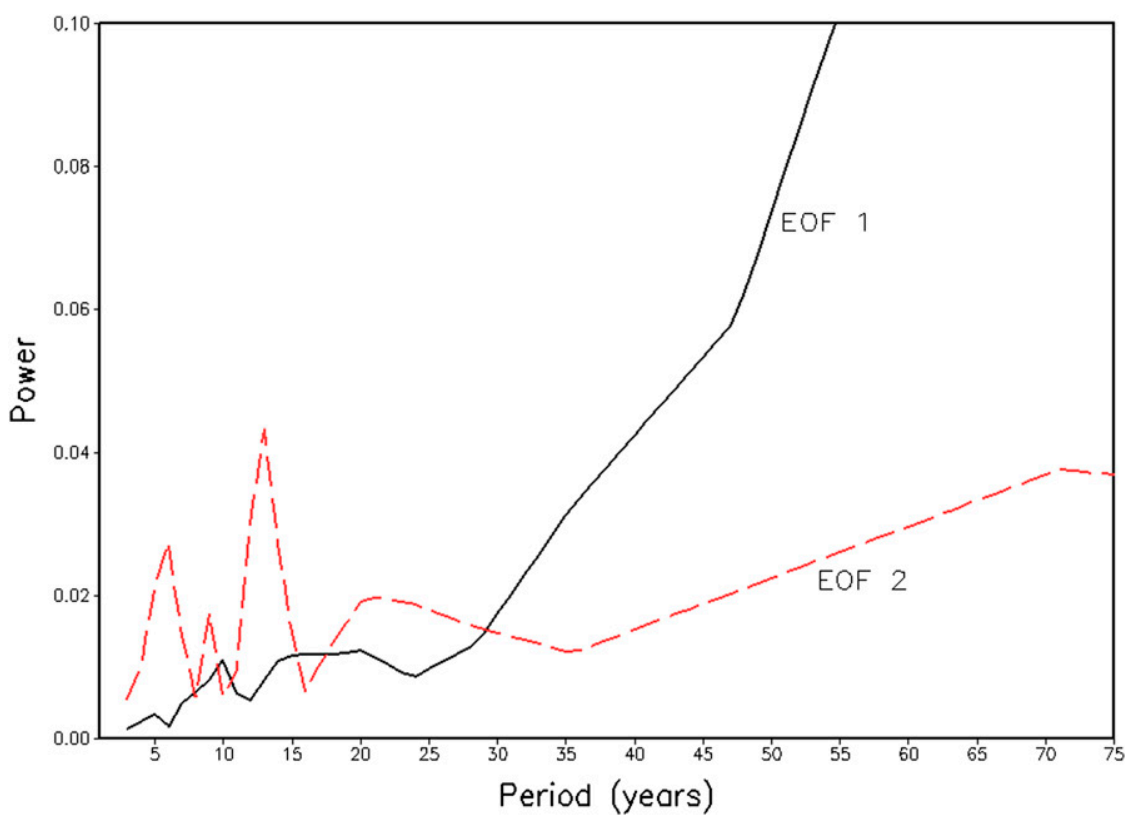

FIG. 8. Spectral analysis of the first two EOF time series. The EOF analysis is based on annual ensemble TPW anomalies.

tendencies in that period (Fig. S1 in the supplemental material). The overall trend is similar to the first EOF trend, although the overall trend for that period is less uniformly positive.

The second main ENSO mode shows a decreasing trend since about 1990. Associated with the strong warm ENSO episode of $1997 / 98$ is a modest positive spike in the mode 2 time series in phase with a large positive spike in mode 1. After that episode there was a long-lasting cool episode, shown by the CPC Niño-3.4 anomaly index discussed earlier. That cool episode is associated with a strong negative spike in the mode 2 time series while the mode 1 time series remained positive. These variations suggest that strong warm episodes can occur if interannual variations in both modes are in phase, but that the general tendency in recent years favors cool episodes. Long-term increases in convective precipitation in the monsoon regions and west Pacific tend to favor an increased frequency of cool ENSO conditions by enhancing Walker cell atmospheric circulations over the Pacific, which will tend to strengthen the normal Pacific trade winds. There is evidence for a strengthening Walker cell from data and model simulations (L'Heureux et al. 2013; Sandeep et al. 2014). These results could be influenced by model biases. For example, Yeh and Kirtman (2007) and Latif and Keenlyside (2009) found that the change in ENSO amplitude with global warming is different in different climate models, although a more recent climate model study by Cai et al. (2014) links more frequent extreme ENSO episodes to global warming. However, the R20C model uses observations of SST and SLP, which should greatly reduce any model biases that could influence our results.

To help confirm our results for the recent period, we computed EOFs of annual RSS satellite-based anomalies, 1988-2012. The resulting first two EOFs (see Fig. S2 in the supplemental material) are similar to smoothed versions of the monthly modes in Fig. 3 and suggest similar multidecadal variations in the recent period. For the $25-\mathrm{yr}$ period, the first annual RSS EOF is dominated by ENSO variations and its time series has a negative tendency. The annual RSS second mode has a multidecadal signal mixed with ENSO, and its time series has a slight positive trend. This general consistency helps to confirm the recent ENSO trend from the reconstructed EOFs.

\section{b. SST annual EOFs}

As a second test we compute comparable EOFs using the extended reconstructed SSTs of Smith et al. (2008). There are many similarities between the TPW and SST annual anomaly variations in both the spatial patterns and time series of the first two modes (Fig. 9). As with the annual TPW EOFs, the first two annual SST EOFs are most important (Table 5). Both TPW and SST annual EOF analyses have global multidecadal variations in the first mode, and the first mode time series have a correlation of 0.95 . Both have ENSO variations in the second mode, and the second mode time series have a correlation of 0.93 . For both SST and TPW, the ENSO mode time series has a negative tendency since the 1980s, a period with good SST sampling. The ENSO time series 


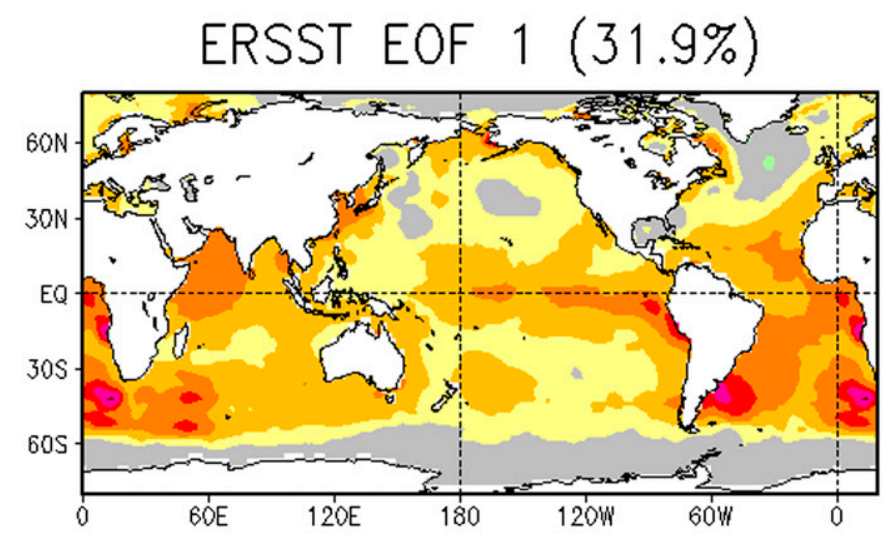

\section{ERSST EOF $2(15.7 \%)$}
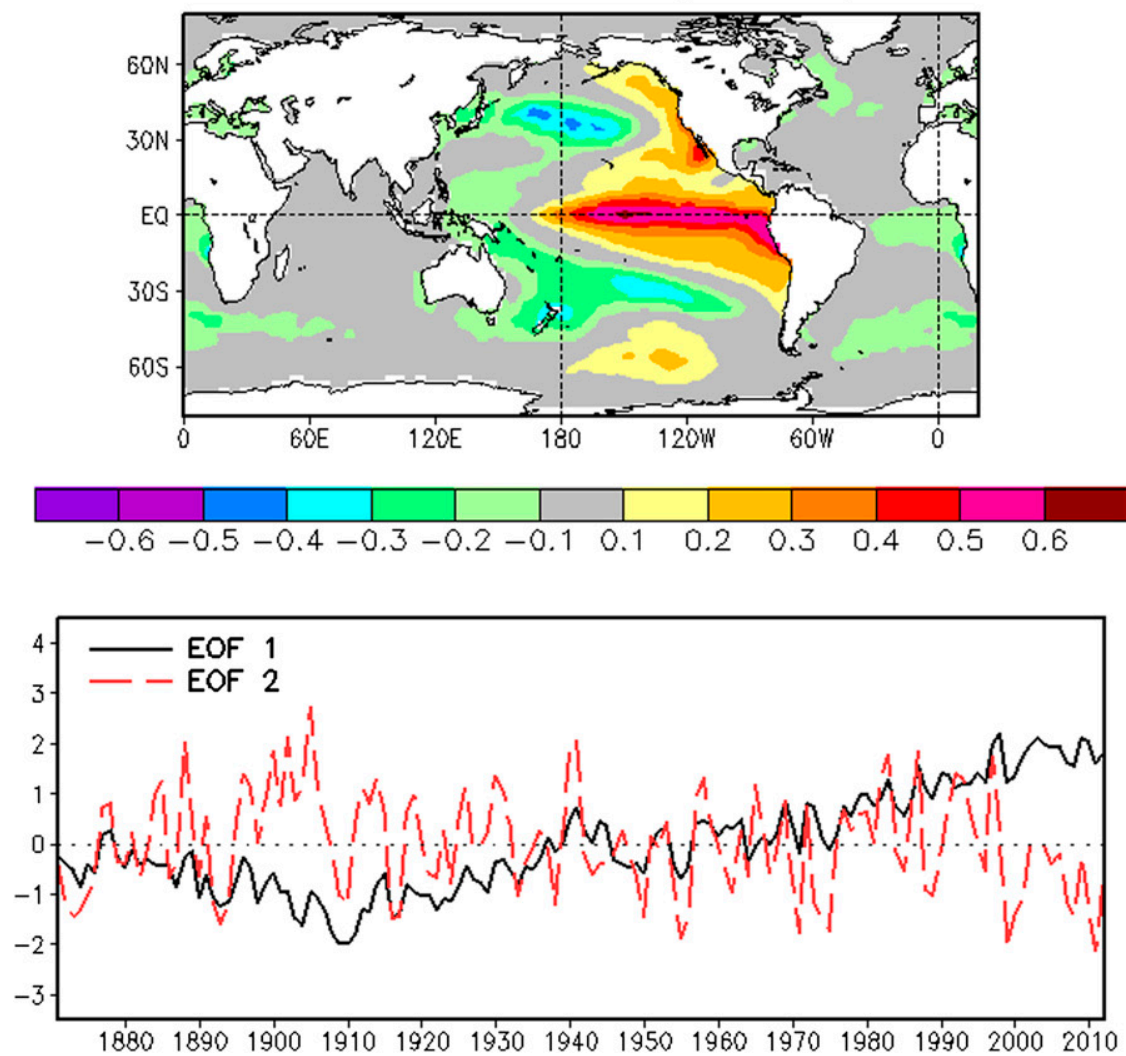

FIG. 9. First two covariance-based EOFs of annual average reconstructed SST anomalies from Smith et al. (2008), 1871-2012. Spatial patterns are shown for (top) EOF 1 and (middle) EOF 2 and (bottom) their time series. Units of time series multiplied by spatial loading values are degrees Celsius.

is also consistent with the eastern equatorial Pacific SST time series from Hansen et al. (2006) in that both show little trend before 1980 and a decreasing long-term mean afterward.

Although there are many similarities, there are also important differences between the annual TPW and SST EOFs. In the multidecadal mode, TPW has strongest variations at low latitudes while for SST there are stronger extratropical multidecadal variations, especially in the Southern Hemisphere. The TPW has a nonlinear sensitivity to SST, as indicated by Eq. (1), and slight changes in tropical SSTs can have a large influence on TPW variations. Thus, TPW variations tend to be much larger in the tropics. The SST variations tend to be large 
TABLE 5. Percent variance accounted for by each of the first 10 EOFs of annual extended reconstructed SST anomalies, 18712012.

\begin{tabular}{ccccccccccc}
\hline \hline EOF & 1 & 2 & 3 & 4 & 5 & 6 & 7 & 8 & 9 & 10 \\
\hline Variance (\%) & 31.9 & 15.7 & 5.2 & 4.4 & 3.6 & 3.5 & 2.2 & 2.2 & 2.0 & 1.8
\end{tabular}

where horizontal gradients are large, such as in the Southern Ocean. Small shifts in gradients can cause large SST changes in those locations. Compared to TPW, the SST tropical Pacific ENSO-mode variations are more concentrated in the equatorial Pacific and farther east toward the region where the thermocline is shallowest and where upwelling normally cools the SST. During warm ENSO episodes that region can be much warmer than normal, while the SST variance in the west Pacific is much less. The TPW ENSO is strongest farther west, where convection anomalies associated with ENSO are stronger. It also has strong variations over a much larger area, apparently reflecting atmospheric teleconnections associated with ENSO. The SST tropical Pacific multidecadal variations are also strongest closer to the equator, apparently reflecting changes in equatorial upwelling, while for TPW the tropical Pacific multidecadal change is strongest just south of the equator, suggesting convergence in that region may be increasing.

The changes in the ENSO mode could be explained by strengthening of the average Pacific trade winds associated with strengthening of the average Walker cell in the region. Since the surface trade winds are critical to containing and concentrating warm waters in the western tropical Pacific Ocean, increasing their strength could cause a prolonged buildup in the western Pacific warm pool. During a longer buildup period, strong trades would produce cool episodes. The longer buildup would favor a strong warm ENSO episode when it is eventually triggered. Since this process will tend to cause more ocean heat storage in the buildup phase, it has been suggested as a possible cause for the recent apparent slowdown in global warming (e.g., Trenberth and Fasullo 2013; England et al. 2014).

Although the idea outlined above explains variations in the first two annual EOFs of TPW and SST, the evidence for it is somewhat mixed. For example, Held and Soden (2006) and Vecchi and Soden (2007) showed that atmospheric models tend to weaken tropical circulations associated with global warming, including the strength of the Walker circulation. However, Gastineau et al. (2009) describe a model in which the descending branch of the Pacific Walker cell strengthens with global warming, producing a stronger cell over the eastern Pacific. That is consistent with Trenberth and Fasullo (2013), who use ECMWF reanalysis output to show increasing Pacific trade winds since 1999, when the trend in the ENSO time series is strongest. The analysis of L'Heureux et al. (2013) also shows an increasing Walker circulation since about 1950, when data are sufficient to evaluate variations. A recent model study by Sandeep et al. (2014) suggests that the background warming in the region is responsible for a generally increasing Pacific Walker circulation, while ENSO episodes weaken the zonal SST gradients and the Walker circulation when they occur, partly masking the background increase.

The recent multidecadal changes in the ENSO mode are similar to PDO variations, but there is essentially no multidecadal ENSO variability for most of the record, and therefore, PDO alone cannot explain ENSO multidecadal changes. The SST analysis in Hansen et al. (2006) show that the east-west equatorial Pacific gradient has increased since 1980, also consistent with our ENSO mode and suggesting that there must be stronger equatorial trade winds in that period. They suggest that the increased gradient can help explain stronger warm ENSO episodes in the recent period.

It is likely that no one mode can fully describe either the global variations or ENSO, as is done in this discussion, and higher EOF modes could be important for describing aspects of both. In addition, EOFs statistically separate processes that may be physically related. In this analysis, for both TPW and SST, the first two modes show opposing changes in the eastern equatorial Pacific. However, the global-mode variations in that region could physically influence the ENSO multidecadal variations (e.g., Fedorov and Philander 2000). The first two EOFs could in part reflect changes in the type and patterns of ENSO variations influenced by global warming. Sorting out the processes more clearly may require studies combining models and observations and is beyond the scope the present study.

\section{c. TPW regressions}

To show differences between individual TPW analyses and the ensemble, we regress the annual superensemble EOF time series from Fig. 7 against annual anomalies from individual analyses. Regression at each point yields maps for each analysis associated with each mode. Here we compare regressions against R20C and $\mathrm{R}$ [WSST]. Although the R20C skill is relatively good, we showed earlier that we can improve it using the superensemble. Among the individual analyses the R[WSST] skill is lowest, and the regressions against it are used to indicate how well it resolves the major TPW variations.

For EOF 1, the R20C regression is very similar to the EOF 1 spatial pattern. In comparison, the R[WSST] regression is more damped and the spatial patterns are 


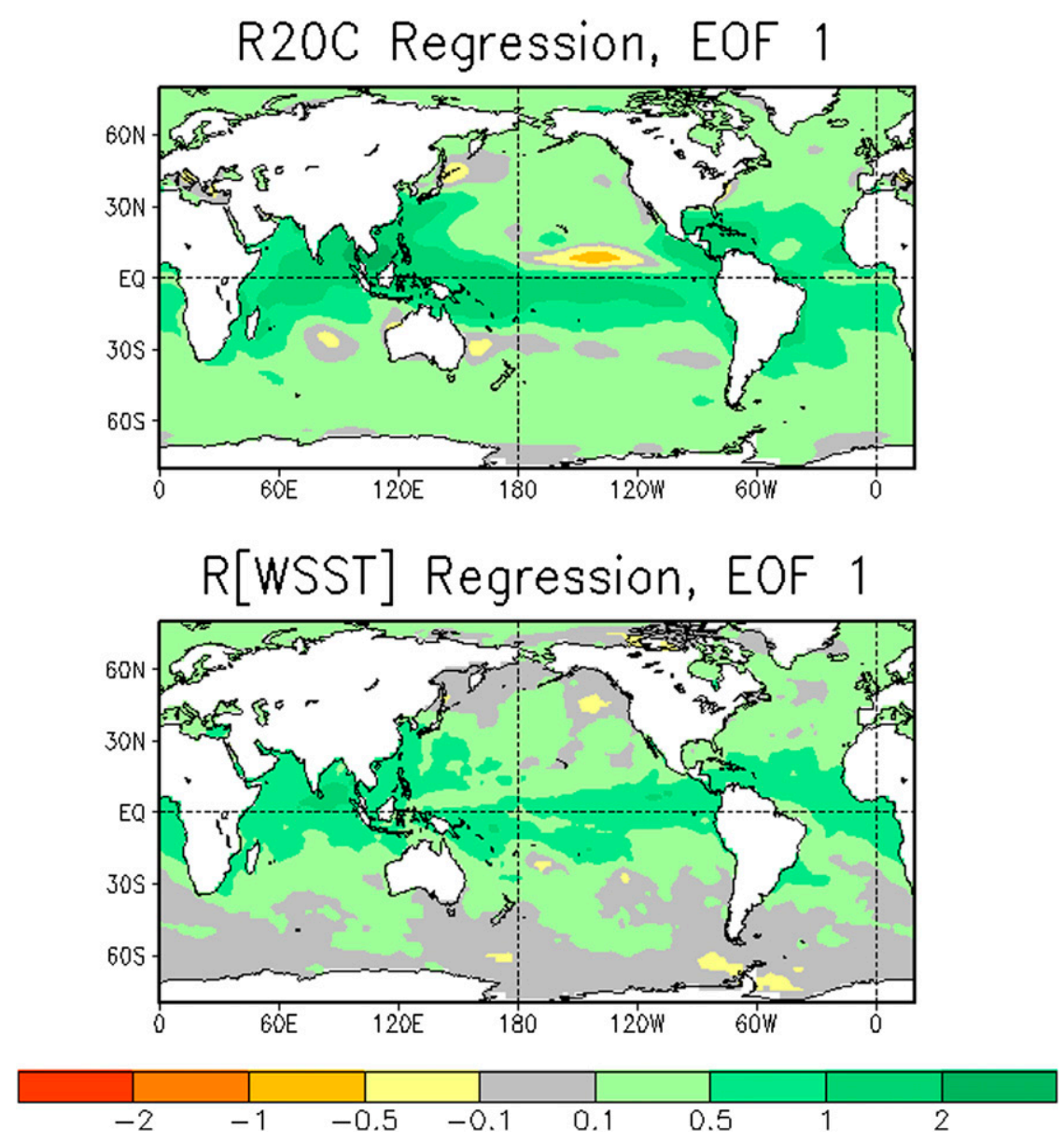

FIG. 10. Regression of individual superensemble inputs against annual TPW EOF 1 time series from Fig. 7. Regression is for annual anomalies from (top) R20C and (bottom) R[WSST] TPW. Units are the same as for the spatial patterns in Fig. 7.

less well defined, but it has a similar overall pattern (Fig. 10). One noticeable difference is that the R[WSST] regression shows that variations in the Pacific are strongest along the equator, while in the $\mathrm{R} 20 \mathrm{C}$ regression they are shifted slightly south. The EOF 2 regression comparisons are similar in the sense that the R20C regressions more closely match the EOF 2 spatial pattern while the R[WSST] regressions are more damped but give a similar pattern (Fig. 11). The R[WSST] regressions more closely resemble the SST EOF patterns, which is understandable considering that WSST is estimated from SST in a fairly simple way. Although most of the superensemble variance is derived from the R20C, the regressions (Figs. 10, 11) indicate that the relatively simple R[WSST] represents much of the large-scale multidecadal and interannual TPW variation.

\section{d. TPW global and annual averages}

Global and annual averages of these TPW analyses (Fig. 12) show that the large-scale means all have much in common. There are two periods with a relatively strong positive trend, roughly 1910-40 and after 1975. Before 1910 there is a slight decreasing trend, and in 1940-75 there is practically no trend. The interannual variations are also similar for all the estimates. As the EOF comparisons indicate, the major TPW variations are closely linked to SST variations. The largest difference is that the R[WSST] analysis tends to be weak compared to R20C. As discussed earlier, all the R[WSST] reconstruction modes are adequately resolved for that period, so differences are not due to inadequate sampling causing modes to be screened out. It is most likely that errors in the WSST anomalies are larger, causing the weights for some of the modes to be damped compared to the weights for $\mathrm{R}[\mathrm{R} 20 \mathrm{C}]$. The WSST errors can be from historical SST errors and from poorer resolution of WSST compared to the more sophisticated R20C analysis. Fully determining causes for the differences is beyond the scope of this study. In any case, the overall positive correlations of the R[WSST] 


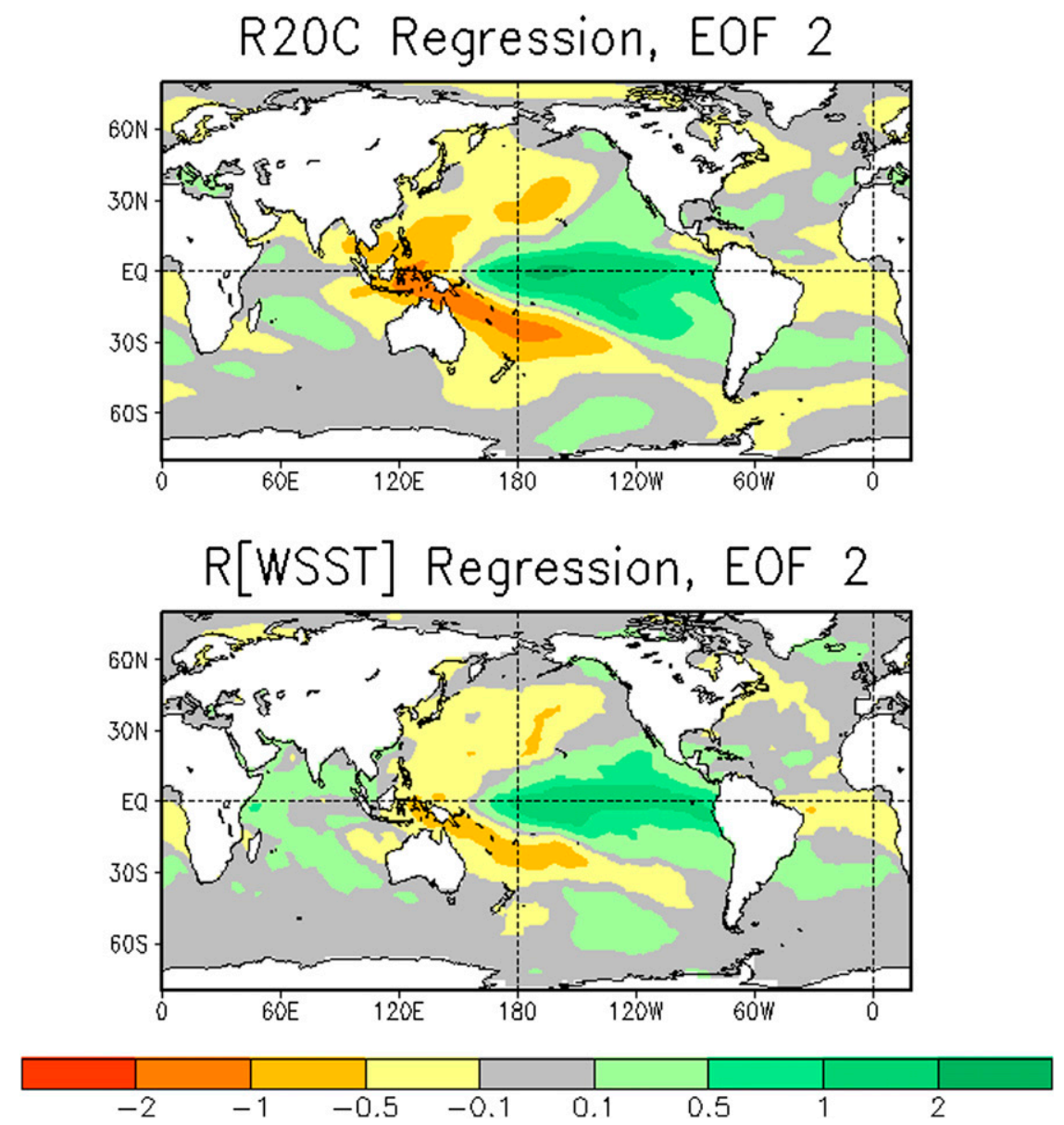

FIG. 11. As in Fig. 10, but for EOF 2 time series from Fig. 7.

analysis with the others indicates that this relatively simple analysis can resolve much of the large-scale TPW variation. That is especially true in regions with SST $>15^{\circ} \mathrm{C}$, where WSST can be directly computed. However, some extratropical variations can also be analyzed through teleconnections in the R[WSST] modes. This reinforces the idea that SST variations may be used to make first-order estimates of large-scale TPW variations, including first-order estimates of future TPW based on SST projections. For example, using the Reynolds et al. (2002) SST analysis, the 19882012 global-mean SST is $18.2^{\circ} \mathrm{C}$. Using that temperature in Eq. (1) yields a TPW estimate of $21.8 \mathrm{~kg} \mathrm{~m}^{-3}$. The SST warming over the twentieth century is roughly $0.7^{\circ} \mathrm{C}$, and using an SST of $17.5^{\circ} \mathrm{C}$ yields a TPW estimate of $20.8 \mathrm{~kg} \mathrm{~m}^{-3}$, or $1 \mathrm{~kg} \mathrm{~m}^{-3}$ lower than in the base period. That is consistent with the TPW global-annual average change over the twentieth century (Fig. 12). Similarly, we can use Eq. (1) to show that if globalmean SST is warmed by $0.7^{\circ} \mathrm{C}$ the mean TPW will increase by about $1.1 \mathrm{~kg} \mathrm{~m}^{-3}$, to $22.9 \mathrm{~kg} \mathrm{~m}^{-3}$. For this modest range of temperature, the change in TPW is nearly linear with temperature. With a larger temperature range, the nonlinear aspect of the relationship between temperature and TPW is more important. For example, if global-mean SST were to increase by $3^{\circ} \mathrm{C}$, then TPW would increase by $5 \mathrm{~kg} \mathrm{~m}^{-3}$. Depending on how the temperature change influences relative humidity, the TPW change could change cloudiness and rainfall.

\section{Summary and conclusions}

We produce a historical TPW analysis with improved skill by statistically combining TPW outputs from an extended dynamic reanalysis (R20C) and a reconstruction based on historical SST. Statistics for the improvements are based on the Remote Sensing Systems (RSS) satellite-based analysis. A high-quality modern analysis such as this is essential for computing statistics. Cross-validation testing is used to evaluate skill and guide the improvements. Here improvements are 


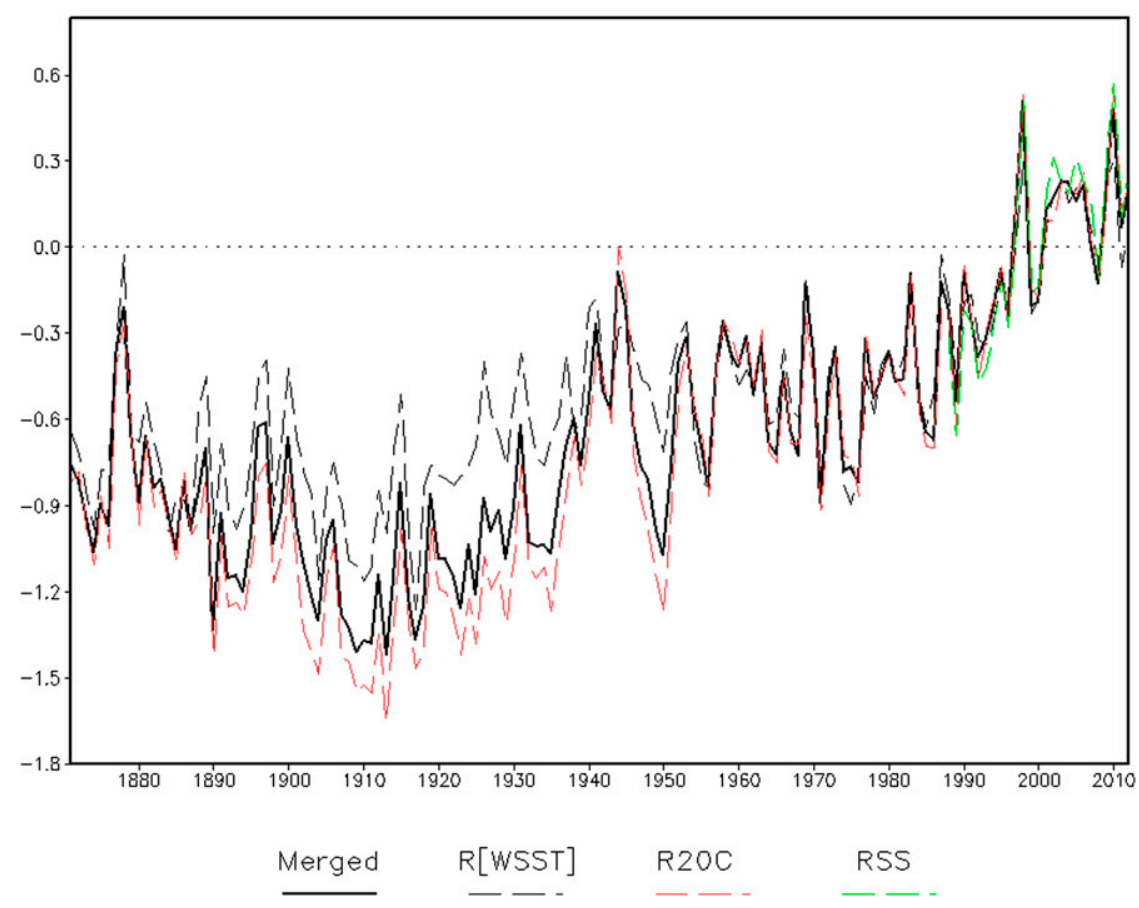

FIG. 12. Global ocean area annual average TPW anomalies from the superensemble (merged, solid black) and the indicated individual historical analyses $\{\mathrm{R}$ [WSST] (dashed black) and R20C (dashed red)\}. The satellite-based RSS analysis is also shown for comparison (dashed green). Units are kilograms per meter squared.

evaluated using correlations with RSS. Compared to the R20C TPW, the cross-validation improvement against unsmoothed RSS is $4 \%$. When RSS is smoothed to filter out small-scale variations that may not be well represented in historical periods, the improvement is $12 \%$. Results suggest that other dynamic reanalysis climate fields could be improved in a similar way. Cross-validation shows that the superensemble approach used here can improve analysis skill even when merging includes analyses with much lower overall skill.

The historical TPW increases with warming global temperatures, consistent with theory. The first EOF of annual reconstructed TPW shows that it increases over most of the global oceans. That increase in TPW is particularly strong in regions such as the western tropical Pacific and Indian Ocean basins, where it may contribute to stronger monsoon rainfall, and around Central America, where it could contribute to enhanced tropical rainfall. The second EOF of annual reconstructed TPW is dominated by ENSO, with little trend over most of the historical period but a negative trend beginning in the 1980s. There is a similar trend in the ENSO mode of annual RSS data for the satellite period, and also in the ENSO mode of annual reconstructed SST data, showing that the trend appears to reflect a real multidecadal ENSO change. That recent trend suggests that warm
ENSO episodes are becoming less frequent, although strong warm ENSO episodes are still possible.

The global-mean oceanic TPW has two periods of increasing trend strongly linked to SST variations, one 1910-40 and the other beginning 1975. For the most recent period the increases are consistent with the RSS and have been discussed by Wentz et al. (2007) and Chung et al. (2014). The study of Chung et al. (2014) shows that the most recent increase is explained by the increase in global temperatures. The strong relationship between SST and TPW shown here makes it likely that the historical TPW variations are also forced by temperature changes. This justifies using Eq. (1) to make firstorder estimates of large-scale TPW changes from temperature. That relationship can help to diagnose TPW and related variations, such as precipitation, as a function of temperature.

This reconstruction can help to better explain the global hydrologic cycle through an improved understanding of long-term changes in TPW over oceans. Potential applications of these data include model validation, climate monitoring, and large-scale climate studies using multiple datasets to evaluate coherent climate variations. The TPW analysis can be particularly useful for helping to identify variations in water vapor source regions since the nineteenth century. 
Acknowledgments. We thank R. Ferraro and W. Yang for useful discussions about TPW and two anonymous reviewers for helpful comments and suggestions. We also thank several centers for making their data easily available for this study, including the TPW data from Remote Sensing Systems, Twentieth Century Reanalysis TPW estimates from the NOAA/Earth System Research Laboratory, OISST data from the National Centers for Environmental Prediction, and historical SST from the Met Office Hadley Centre (www.metoffice.gov.uk/ hadobs). This project is supported in part by the Cooperative Institute for Climate and Satellites (CICS), NOAA Grant NA09NES4400006, at the ESSIC, University of Maryland, College Park. The contents of this paper are solely the opinions of the authors and do not constitute a statement of policy, decision, or position on behalf of NOAA or the U.S. Government.

\section{APPENDIX A}

\section{The Reconstruction Method}

The reconstruction method is briefly described in this appendix. Some additional details are given by Smith et al. $(1996,1998)$. The method analyzes sparsely sampled historical anomalies by fitting them to a set of EOF spatial modes. Here covariance-based EOFs are used. If correlation-based EOFs are used then the data fit to the modes should be normalized. The EOFs are computed using spatially complete RSS monthly anomalies over 1988-2012.

The anomalies are fit to the set of modes to produce the set of weights that minimize the mean-squared error (MSE) of the analysis as measured against the available data. The analysis is defined as

$$
F(x, t)=\sum_{m=1}^{M} \psi_{m}(x) w_{m}(t) .
$$

Here $\psi_{m}(x)$ is the EOF mode $m$ eigenvector at spatial location $x$, and $w_{m}(t)$ is the optimal mode weight for time $t$. The total number of modes used is $M$. The best-fit weights for the modes are computed separately for each time. The spatial MSE is defined at each time as

$$
E^{2}=\sum_{x=1}^{K}[D(x, t)-F(x, t)]^{2} \delta(x) \cos \varphi(x) .
$$

The data anomalies being fit to the modes are $D(x, t)$; $\delta(x)$ is a delta function equal to 1 if there is sampling at location $x$ and 0 otherwise; and $\varphi(x)$ is the latitude of location $x$, used as an area-weighting factor for the gridded data. The total number of spatial locations is $K$. The minimum MSE is found by solving

$$
\frac{\partial E^{2}}{\partial w_{m}}=0, \text { for } m=1,2, \ldots, M
$$

By substituting Eqs. (A1) and (A2) into Eq. (A3), it can be shown that the weights are computed by solving the system of equations

$$
\begin{aligned}
\sum_{m=1}^{M} & {\left[w_{m} \sum_{x=1}^{K} \psi_{m}(x) \psi_{n}(x) \delta(x) \cos \varphi(x)\right] } \\
& =\sum_{x=1}^{K} D(x, t) \psi_{n}(x) \delta(x) \cos \varphi(x), \text { for } m=1,2, \ldots, M
\end{aligned}
$$

The system of equations from Eq. (A4) is solved numerically to yield the optimal set of weights for reconstruction.

Because of the sparse historical sampling, some modes may not always be adequately sampled. A badly sampled mode could inflate random errors in the historical data, so modes are screened out of the analysis when their sampling is not adequate. When modes are adequately sampled, the random errors are almost completely filtered out of the analysis since random variations will not match the coherent variations represented by the modes. Sampling of each mode is described using the fraction of the mode variance sampled by the available data,

$$
f_{m}=\frac{\sum_{x=1}^{K} \psi_{m}^{2}(x) \delta(x) \cos \varphi(x)}{\sum_{x=1}^{K} \psi_{m}^{2}(x) \cos \varphi(x)} .
$$

If this fraction falls below a critical fraction for the mode $m$, then that mode is excluded from the reconstruction. Here a critical fraction of 0.05 is used, which we have found to be sufficient to filter out almost all noise while minimizing analysis damping.

\section{APPENDIX B}

\section{Time Filtering and Degrees of Freedom}

Here we briefly discuss time filters tested for separating short- and long-period variations. Here shortperiod variations are those with frequencies of less than about $5 \mathrm{yr}$. There are many types of filters that could potentially be used for this purpose. Testing was done to evaluate the main properties of a few common filters. In addition, a method for estimating the reduction of degrees of freedom in filtered data is also discussed. 
For testing, we generate a 101-point time series to simulate an annual climate time series for a long period. The time series is a linear combination of four separate time series: 1) a linear trend with a mean of 0 and a slope of 1 per $101 \mathrm{yr}, 2)$ a sine wave with a period of $10 \mathrm{yr}$ and an amplitude of 1,3 ) a sine wave with a period of $3 \mathrm{yr}$ and an amplitude of 1, and 4) Gaussian random noise with a mean of 0 and a standard deviation of 0.5 .

Testing is done for two binomial filters, one with a length of $7 \mathrm{yr}$ and the other with a length of $11 \mathrm{yr}$. The test demonstrates that both binomial filters work about equally well. Using either filter, the random component is removed and the trend and $10-\mathrm{yr}$ variations are retained. However, in both the 3-yr cycle modifies the amplitude of the 10-yr cycle. Since there is little difference, we will use the seven-point binomial filter to separate short-period from multidecadal variations.

The number of degrees of freedom is needed for significance testing, so it is useful to know the reduction in the number of degrees of freedom caused by filtering. We can roughly estimate that reduction using the relationship between the variance of a mean $\sigma_{A}^{2}$ and the variance of the individual variables $x_{i}$, each with a variance $\sigma_{i}^{2}$. In this estimate we make a few assumptions to simplify the estimation. If we use equal weighting of the $n$ variables, then

$$
\sigma_{A}^{2}=\frac{1}{n^{2}}\left(\sum_{i=1}^{n} \sigma_{i}^{2}+\sum_{j=2}^{n} \sum_{i=1}^{j-1} \sigma_{i} \sigma_{j} r_{i, j}\right) .
$$

Here $r_{i, j}$ is the correlation between the individual variables. If we assume that the correlations can all be estimated by their average $r_{a}$, and that the individual variances can be estimated by their average $\sigma_{a}^{2}$, then Eq. (B1) simplifies to

$$
\sigma_{A}^{2}=\frac{\sigma_{a}^{2}}{n}\left[1+(n-1) r_{a}\right]
$$

Here $x_{i}$ represents values of the time series being filtered and $r_{a}$ accounts for all correlations between variables, including autocorrelation. Assuming that this correlation is constant makes this a crude estimate of the variance of the mean, used here only to make an estimate of the reduction in degrees of freedom.

Another estimate of the variance of the mean is $\sigma_{A}^{2}=\sigma_{a}^{2} / n_{*}$, where $n_{*}$ is the number of degrees of freedom (e.g., Kagan 1979). Using this identity, we can write

$$
n_{*}=\frac{n}{1+(n-1) r_{a}} .
$$

Note that if variables are uncorrelated then $n_{*}=n$, while if they are perfectly correlated then $n_{*}=1$. In our case $n$ can represent the length of the time series, and the degrees of freedom estimate is for the number of independent samples in the time series. The average correlation between variables is related to the filtering. In general, the greater the filtering, the higher the average correlation will be.

Now consider the estimated number of degrees of freedom for an unfiltered time series $n_{*}(0)$ and for a filtered time series $n_{*}(f)$. The ratio is

$$
\frac{n_{*}(f)}{n_{*}(0)}=\frac{1+(n-1) r_{0}}{1+(n-1) r_{f}} .
$$

The limit of the ratio as $n \rightarrow \infty$ is $r_{0} / r_{f}$, which gives a rough estimate of the change in degrees of freedom from filtering. For example, if the measured average correlation before filtering is 0.3 and after filtering we find it is 0.6 , then we know that the filtering reduces the degrees of freedom by roughly 0.5 . By definition there can never be less than 1 degree of freedom, so an estimated reduction to less than 1 should be set to 1 .

\section{REFERENCES}

Biondi, F., A. Gershunov, and D. R. Cayan, 2001: North Pacific decadal climate variability since 1661 . J. Climate, 14, 5-10, doi:10.1175/1520-0442(2001)014<0005:NPDCVS > 2.0.CO;2.

Cai, W., and Coauthors, 2014: Increasing frequency of extreme El Niño events due to greenhouse warming. Nat. Climate Change, 4, 111-116, doi:10.1038/nclimate2100.

Chung, E.-S., B. Soden, B. J. Sohn, and L. Shi, 2014: Uppertropospheric moistening in response to anthropogenic warming. Proc. Natl. Acad. Sci., 111, 11636-11641, doi:10.1073/ pnas.1409659111.

Compo, G. P., and Coauthors, 2011: The Twentieth Century Reanalysis Project. Quart. J. Roy. Meteor. Soc., 137, 1-28, doi:10.1002/qj.776.

Davis, R. E., 1976: Predictability of sea surface temperature and sea level pressure anomalies over the North Pacific Ocean. J. Phys. Oceanogr., 6, 249-266, doi:10.1175/1520-0485(1976)006<0249: POSSTA $>2.0 . \mathrm{CO} ; 2$.

Dong, L., and T. Zhou, 2014: The formation of the recent cooling in the eastern tropical Pacific Ocean and the associated climate impacts: A competition of global warming, IPO, and AMO. J. Geophys. Res. Atmos., 119, 11272-11287, doi:10.1002/ 2013JD021395.

England, M. H., and Coauthors, 2014: Recent intensification of wind-driven circulation in the Pacific and the ongoing warming hiatus. Nat. Climate Change, 4, 222-227, doi:10.1038/ nclimate2106.

Fedorov, A. V., and S. G. Philander, 2000: Is El Niño changing? Science, 288, 1997-2002, doi:10.1126/science.288.5473.1997.

Gastineau, G., L. Li, and H. Le Treut, 2009: The Hadley and Walker circulation changes in global warming conditions described by idealized atmospheric simulations. J. Climate, 22, 3993-4013, doi:10.1175/2009JCLI2794.1.

Glahn, H. R., 1985: Statistical weather forecasting. Probability, Statistics, and Decision Making in Atmospheric Sciences, A. H. Murphy and R. W. Katz, Eds., Westview Press, 545 pp. 
Hansen, J., M. Sato, R. Ruedy, K. Lo, D. W. Lea, and M. MedinaElizade, 2006: Global temperature change. Proc. Natl. Acad. Sci., 103, 14288-14 293, doi:10.1073/pnas.0606291103.

Hartmann, D. L., and Coauthors, 2014: Observations: Atmosphere and surface. Climate Change 2013: The Physical Science Basis, T. F. Stocker et al., Eds., Cambridge University Press, 159254.

Held, I., and B. J. Soden, 2006: Robust response of the hydrological cycle to global warming. J. Climate, 19, 5686-5699, doi:10.1175/ JCLI3990.1.

Kagan, R. L., 1979: Averaging Meteorological Fields. Gidrometeoizdat, $212 \mathrm{pp}$.

Kennedy, J. J., N. A. Rayner, R. O. Smith, M. Saunby, and D. E. Parker, 2011a: Reassessing biases and other uncertainties in sea surface temperature observations measured in situ since 1850: 1. Measurement and sampling uncertainties. J. Geophys. Res., 116, D14103, doi:10.1029/2010JD015218.

,,,$--- \ldots$, and — 2011b: Reassessing biases and other uncertainties in sea surface temperature observations measured in situ since 1850: 2. Biases and homogenization. J. Geophys. Res., 116, D14104, doi:10.1029/2010JD015220.

Kidder, S. Q., and A. S. Jones, 2007: A blended satellite total precipitable water product for operational forecasting. J. Atmos. Oceanic Technol., 24, 74-81, doi:10.1175/JTECH1960.1.

Latif, M., and N. S. Keenlyside, 2009: El Niño/Southern Oscillation response to global warming. Proc. Natl. Acad. Sci. USA, 106, 20 578-20 583, doi:10.1073/pnas.0710860105.

L'Heureux, M. L., S. Lee, and B. Lyon, 2013: Recent multidecadal strengthening of the Walker circulation across the tropical Pacific. Nat. Climate Change, 3, 571-576, doi:10.1038/ nclimate 1840 .

Li, H., and B. D. Chen, 2005: The evolution of precipitable water associated with the Asian and Australian monsoons as revealed from MODIS/SSMI, ECMWF and NCEP reanalysis data sets. Geophys. Res. Lett., 32, L10811, doi:10.1029/ 2005GL022570.

Lin, R., T. Zhou, and Y. Qian, 2014: Evaluation of global monsoon precipitation changes based on five reanalysis datasets. J. Climate, 27, 1271-1289, doi:10.1175/JCLI-D-13-00215.1.

Liu, H., T. Zhou, Y. Zhu, and Y. Lin, 2012: The strengthening East Asia summer monsoon since the early 1990s. Chin. Sci. Bull., 57, 1553-1558, doi:10.1007/s11434-012-4991-8.

Mantua, N. J., S. R. Hare, Y. Zhang, J. M. Wallace, and R. C Francis, 1997: A Pacific interdecadal climate oscillation with impacts on salmon production. Bull. Amer. Meteor. Soc., 78,1069-1079, doi:10.1175/1520-0477(1997)078<1069: APICOW $>2.0 . \mathrm{CO} ; 2$.

Mears, C. A., B. D. Santer, F. J. Wentz, K. E. Taylor, and M. F. Wehner, 2007: Relationship between temperature and precipitable water changes over tropical oceans. Geophys. Res. Lett., 34, L24709, doi:10.1029/2007GL031936.

North, G. R., T. L. Bell, R. F. Cahalan, and F. J. Moeng, 1982: Sampling errors in estimation of empirical orthogonal functions. Mon. Wea. Rev., 110, 699-706, doi:10.1175/ 1520-0493(1982)110<0699:SEITEO > 2.0.CO;2.

Panofsky, H. A., and G. W. Brier, 1958: Some Applications of Statistics to Meteorology. Pennsylvania State University Press, 224 pp.

Remote Sensing Systems, cited 2014: Monthly mean total precipitable water 1-deg monthly climate product. Version-7 release-0 TPW CDR, Remote Sensing Systems, Santa Rosa, CA. [Available online at http://www.remss.com/measurements/ atmospheric-water-vapor/tpw-1-deg-product.]

Reynolds, R. W., N. A. Rayner, T. M. Smith, D. C. Stokes, and W. Wang, 2002: An improved in situ and satellite SST analysis. J. Climate, 15, 1609-1625, doi:10.1175/1520-0442(2002)015<1609: AIISAS $>2.0 . \mathrm{CO} ; 2$.

Sandeep, S., F. Stordal, P. D. Sardeshmukh, and G. P. Compo, 2014: Pacific Walker Circulation variability in coupled and uncoupled climate models. Climate Dyn., 43, 103-117, doi:10.1007/s00382-014-2135-3.

Smith, T. M., R. W. Reynolds, and C. F. Ropelewski, 1994: Optimal averaging of seasonal sea surface temperatures and associated confidence intervals (1860-1989). J. Climate, 7, 949-964, doi:10.1175/1520-0442(1994)007<0949:OAOSSS >2.0.CO;2.

,,$- \ldots$, R. E. Livezey, and D. C. Stokes, 1996: Reconstruction of historical sea surface temperatures using empirical orthogonal functions. J. Climate, 9, 1403-1420, doi:10.1175/ 1520-0442(1996)009<1403:ROHSST $>2.0 . C O ; 2$.

— R. E. Livezey, and S. S. P. Shen, 1998: An improved method for analyzing sparse and irregularly distributed SST data on a regular grid: The tropical Pacific Ocean. J. Climate, 11, 1717-1729, doi:10.1175/1520-0442(1998)011<1717: AIMFAS $>2.0 . \mathrm{CO} ; 2$.

R. W. Reynolds, T. C. Peterson, and J. Lawrimore, 2008: Improvements to NOAA's historical merged land-ocean surface temperature analysis (1880-2006). J. Climate, 21, 2283-2296, doi:10.1175/2007JCLI2100.1.

Stephens, G. L., 1990: On the relationship between water vapor over oceans and sea surface temperature. J. Climate, 3, 634-645, doi:10.1175/1520-0442(1990)003<0634:OTRBWV>2.0.CO;2.

Sudradjat, A., R. R. Ferraro, and M. Fiorino, 2005: A comparison of total precipitable water between reanalyses and NVAP. J. Climate, 18, 1790-1807, doi:10.1175/JCLI3379.1.

Trenberth, K. E., and J. Fasullo, 2013: An apparent hiatus in global warming? Earth's Future, 1, 19-32, doi:10.1002/2013EF000165. - , and L. Smith, 2005: Trends and variability in columnintegrated atmospheric water vapor. Climate Dyn., 24, 741758, doi:10.1007/s00382-005-0017-4.

, _— , and J. Mackaro, 2011: Atmospheric moisture transports from ocean to land and global energy flows in reanalyses. J. Climate, 24, 4907-4924, doi:10.1175/2011JCLI4171.1.

Vecchi, G., and B. J. Soden, 2007: Global warming and the weakening of tropical circulation. J. Climate, 20, 4316-4340, doi:10.1175/JCLI4258.1.

Wang, H., and K. M. Lau, 2006: Atmospheric hydrological cycle in the tropics in twentieth century coupled climate simulations. Int. J. Climatol., 26, 655-678, doi:10.1002/joc.1279.

Wentz, F. J., L. Ricciardulli, K. Hilburn, and C. Mears, 2007: How much more rain will global warming bring? Science, 317, 233235, doi:10.1126/science.1140746.

Xue, Y., T. M. Smith, and R. W. Reynolds, 2003: Interdecadal changes of 30-yr SST normal during 1871-2000. J. Climate, 16, 1601-1612, doi:10.1175/1520-0442-16.10.1601.

Yeh, S.-W., and B. P. Kirtman, 2007: ENSO amplitude changes due to climate change projections in different coupled models. J. Climate, 20, 203-217, doi:10.1175/JCLI4001.1.

Zhang, L., and T. Zhou, 2011: An assessment of monsoon precipitation changes during 1901-2001. Climate Dyn., 37, 279296, doi:10.1007/s00382-011-0993-5. 